A NEW COMPUTATIONAL MODEL TO AUGMENT THE DESIGN OF MICROFLUIDIC SEPARATIONS: ELECTRIC FIELD ASSISTED, HYDRODYNAMIC CHROMATOGRAPHY

\author{
A Thesis \\ presented to \\ the Faculty of California Polytechnic State University, \\ San Luis Obispo
}

\author{
In Partial Fulfillment \\ of the Requirements for the Degree \\ Master of Science in Biomedical Engineering \\ by \\ Jeffrey Dean Wells \\ June 2012
}


(C) 2012

Jeffrey Wells

ALL RIGHTS RESERVED 


\section{COMMITTEE MEMBERSHIP}

TITLE:

A NEW COMPUTATIONAL MODEL TO AUGMENT THE DESIGN OF MICROFLUIDIC SEPARATIONS: ELECTRIC FIELD ASSISTED, HYDRODYNAMIC CHROMATOGRAPHY

AUTHOR:

Jeffrey Dean Wells

DATE SUBMITTED:

June 2012

COMMITTEE CHAIR:

David Clague, $\mathrm{PhD}$

Associate Professor, Biomedical and General Engineering

California Polytechnic State University, San Luis Obispo

COMMITTEE MEMBER: $\quad$ Scott Hazelwood, PhD

Associate Professor, Biomedical and General Engineering

California Polytechnic State University, San Luis Obispo

COMMITTEE MEMBER: Dan Walsh, PhD

Professor, Biomedical and General Engineering

California Polytechnic State University, San Luis Obispo 


\title{
ABSTRACT \\ A NEW COMPUTATIONAL MODEL TO AUGMENT THE DESIGN OF MICROFLUIDIC SEPARATIONS: ELECTRIC FIELD ASSISTED, HYDRODYNAMIC CHROMATOGRAPHY
}

\author{
Jeffrey Wells
}

This project encompasses the implementation of a computational model to simulate the microfluidic separation of like-charged particles in a continuous flow environment. By accomplishing this task the model can be used to optimize future fractionations by tailoring the process parameters to the properties of the target particles. The primary goal of this project is to develop a vectorized code within Matlab® that captures a sufficient quantity of the physics in separations to assist with the optimization and design of microfluidic systems.

This project differs from other computational models in that it utilizes a personal computer to run the simulation in an optimized format rather than utilizing a highly parallelized system for the computing. Based on previous literature from computational models of fluid-particle systems a model was developed to simulate the separation process. Computational experiments of separation processes were conducted with this model to validate the simulation and to investigate the impacts of microfluidic fractionation parameters on the purity and yield of like charged particles in a continuous flow environment. By adapting the input parameters the separation results can be customized for the particles in the sample. The implementation and use of this this model can improve the efficiency of separation processes.

Keywords: Brownian Dynamic, Microfluidic, Separation, Computational, Simulation, Model 


\section{ACKNOWLEDGMENTS}

I would like to first thank Dr. David Clague for his support and guidance; without his assistance this thesis would not have been possible. I would additionally like to thank my committee members, Dr. Walsh and Dr. Hazelwood, for their assistance in the review process and defense of the thesis. Finally I would like to thank my family for their endless love and support throughout my education. 


\section{TABLE OF CONTENTS}

LIST OF TABLES.......................................................................................................................................... viii

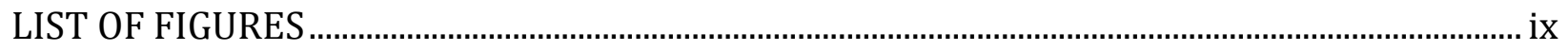

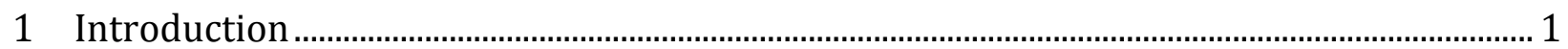

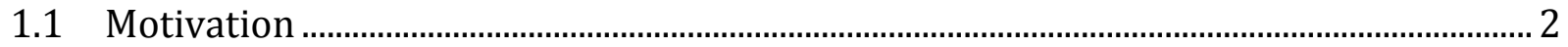

1.2 Clinical Diagnostics............................................................................................................ 2

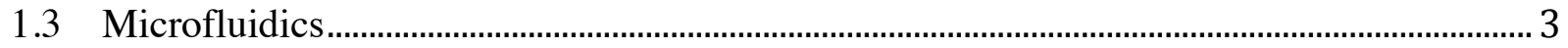

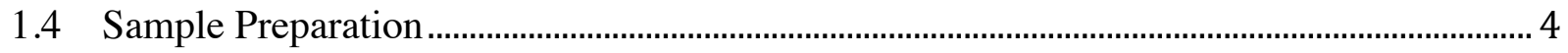

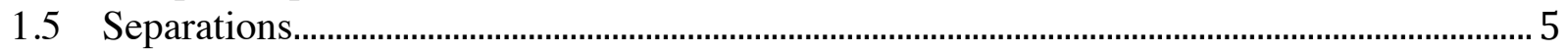

1.5.1 Continuous Flow Separations ............................................................................................ 8

1.5.2 Hydrodynamic Chromatography ……………….............................................................

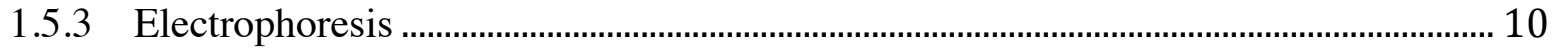

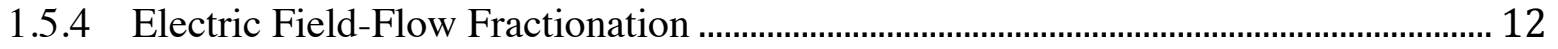

1.6 Separation of like charged species.......................................................................................... 14

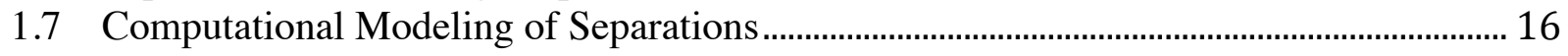

1.7.1 Multi Particle Interactions............................................................................................... 16

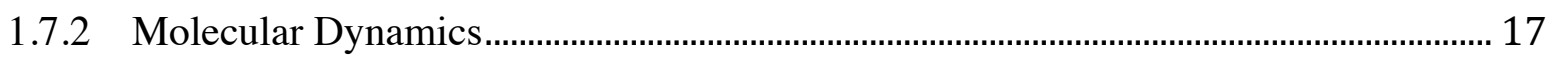

1.7.3 Brownian Dynamics................................................................................................... 17

1.7.4 Lattice Models ....................................................................................................... 18

1.7.5 Direct Simulation of Monte Carlo................................................................................ 18

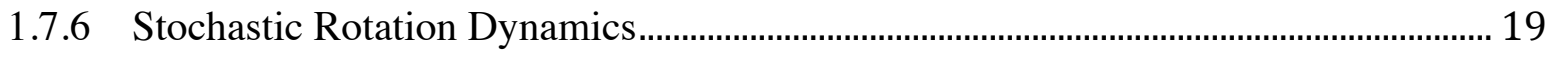

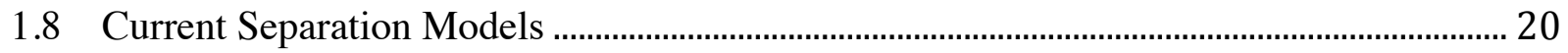

1.9 Project Overview ............................................................................................................... 21

2 Model Development

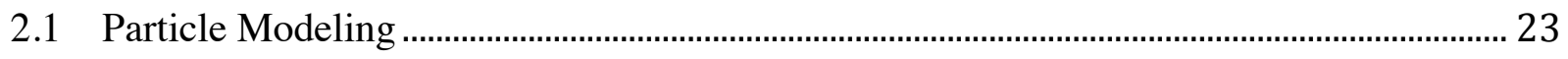

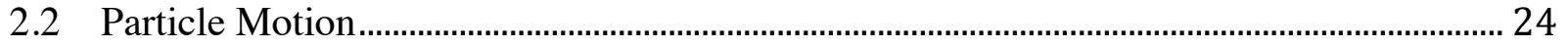

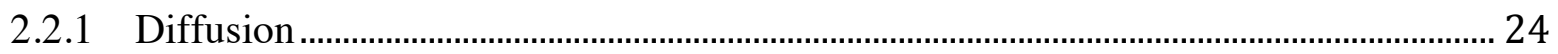

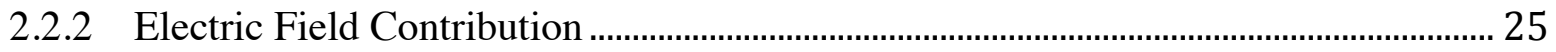

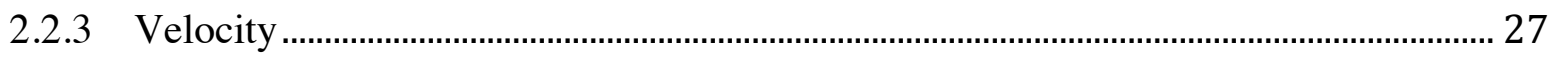

2.3 Particle Interactions ………………………………………..................................................... 29

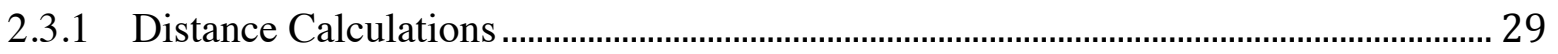

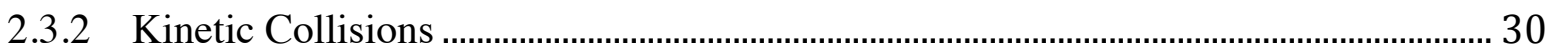

2.3.3 DLVO Interactions ................................................................................................ 32

2.4 Parameters.................................................................................................................... 34

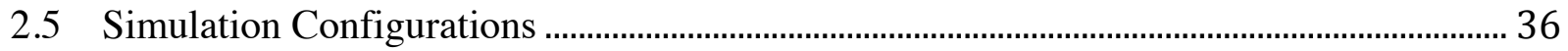

2.6 Limitations ................................................................................................................ 38 


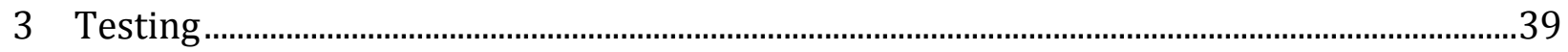

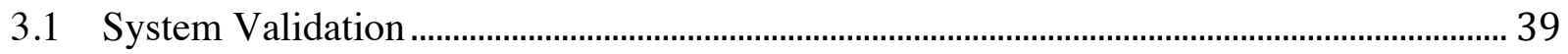

3.1.1 Diffusive Motion.................................................................................................................... 39

3.1.2 Electric Field Motion.................................................................................................... 40

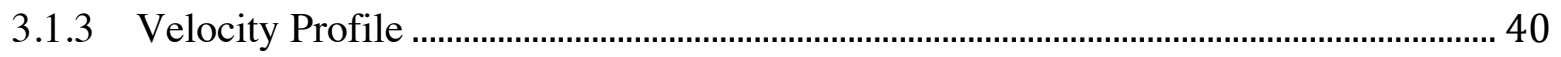

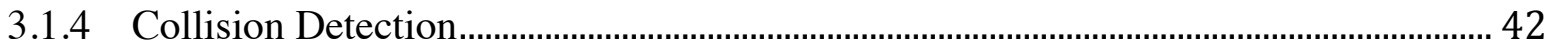

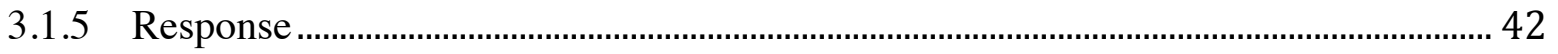

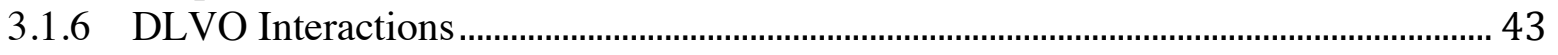

3.2 Simulation of Microfluidic Separation................................................................................... 44

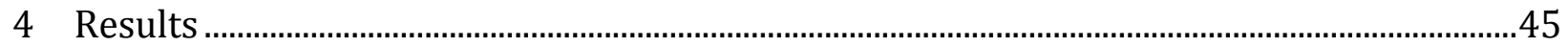

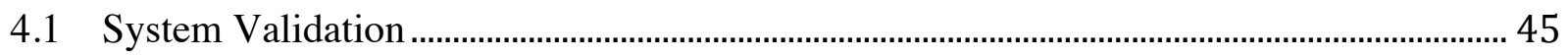

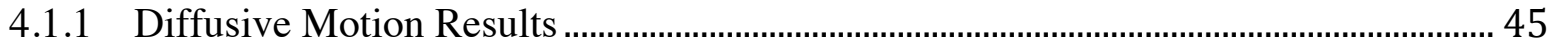

4.1.2 Electric Field Motion Results ..................................................................................... 47

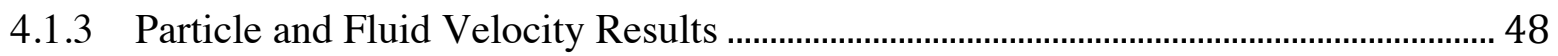

4.1.4 Collision Detection Results ..................................................................................... 49

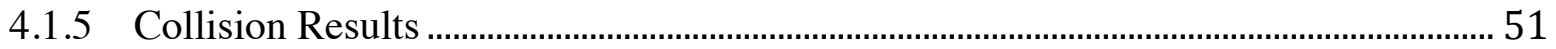

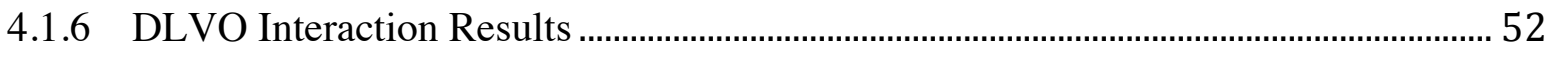

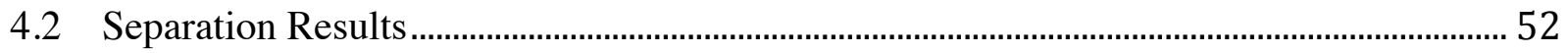

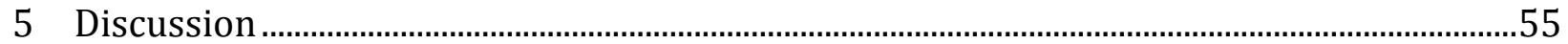

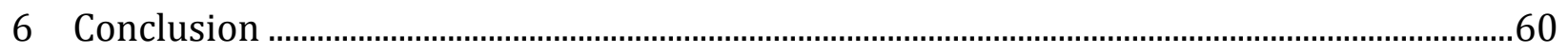

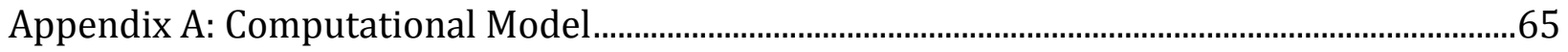

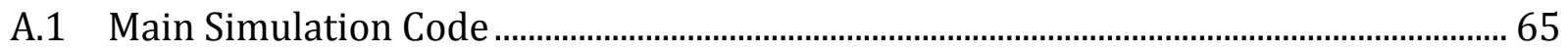

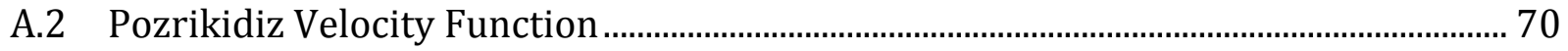

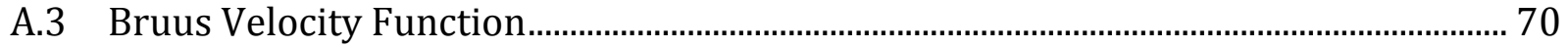

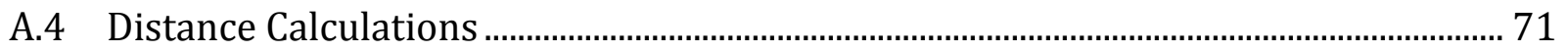

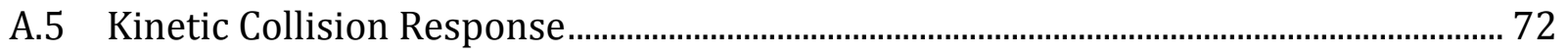

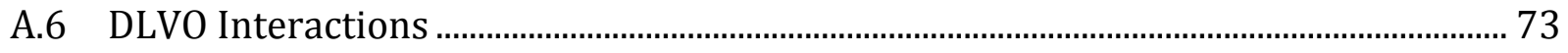




\section{LIST OF TABLES}

Table 1 - Coordinates of particles evaluated in the velocity profile testing. The channel these points reside in is $10 \mathrm{~mm}$ wide and a height of $20 \mathrm{~mm}$.

Table 2 - Particle locations and radii necessary for distance calculations. The Debye length is used to determine whether particles are within the DLVO interaction range..

Table 3 - Kinetic collision input data. The input velocity and mass of interacting particles are required in order to calculate the results from a collision.

Table 4 - Input data for calculating DLVO potentials. The gamma values are specific to the interacting particles; the remaining values relate to fluid properties or are universal constants

Table 5 - Particle properties input for this simulation

Table 6 - Variable elements in this model; 3 flow rates were tested with 11 applied voltages.

All other input values remained static during the simulation

Table 7 - Comparison of the distances traveled as a function of the applied electric field for the simulated and theoretical calculations

Table 8 - Particle coordinates and resulting velocities in the $\mathrm{x}$-direction after calculation with the solutions by Bruus and Pozrikidis. The flow channel was $10 \mathrm{~mm}$ in width and $20 \mathrm{~mm}$ in height.

Table 9 - Indices of particles interacting through collisions or DLVO potentials, based on the particle locations and radii in Table 2.

Table 10 - Pairwise distance between all particles in Table 2. The rows and columns refer to the particle indices.

Table 11 - Particle motion resulting from simulated collision interactions. The colliding pairs of particles leading to these results were 2-6, and 3-7.

Table 12 - Calculated particle motion resulting from collision interactions of particles 2-6, and $3-7$.

Table 13 - The calculated and simulated DLVO potential between interacting particles is shown in the table above. The interaction is attractive if the energy is negative and repulsive if the energy is positive. 


\section{LIST OF FIGURES}

Figure 1 - Compilation of images from a clinical diagnostic laboratory. The upper left displays a technician loading a centrifuge with vials of biologic samples. The upper right shows two technicians viewing results from a test. In the bottom right, two individuals examine a culture dish. The bottom left shows a technician working with sharps

Figure 2 - Diagram of the modular analytic process. The boxes on the ends correspond to the generic input sample and the information gained from it. The four cylindrical steps in the middle follow the sample from the raw state through pre-treatment and processing, after which it is analyzed and interpreted.

Figure 3 - Comparison of batch flow separations with continuous flow systems. a) Drawing of a batch flow fractionation device and the elution profile for separated species. b) Diagram of a continuous flow system and the elution profile of its separated species.

Figure 4 - Conceptual drawing of Hydrodynamic Chromatography. The large particle is limited to the high flow region of the channel while the small particle is able to acces slower flow areas. The diffusion of species results in a higher large particle average velocity than the small particles, leading to elution of the large particles first.

Figure 5 - Conceptual drawing of the three modes of electric Field Flow Fractionation. Left) The normal mode, a low force and diffusive motion of particles drive the separation. Middle) Steric mode, a high force and hydrodynamic effects drive the fractionation. Right) Hyperlayer mode, high flow rate and force lift and separate the particles by hydrodynamic forces

Figure 6 - Sketch of the velocity profile for laminar flow through a rectangular channel. a) Cross sectional view of the velocity distribution looking in the direction of flow; velocity is constant around the topographic tracings with higher velocities in the center and low velocity near the channel walls. b) Cross section looking across the flow in the z-direction. c) Cross section of the flow looking from the y-direction [44].

Figure 7 - Plot of DLVO Interaction Potential based on van der Waals attraction and electric double layer repulsion energies. At small separation distances interacting particles experience an attractive force; however at greater separations this becomes a repulsive potential. When the separation distance becomes sufficiently large the interaction potential approaches zero; this cutoff distance is typically 1 Debye length.

Figure 8 - Block diagram of the simulation algorithm. The model proceeds from top to bottom, looping from separation evaluation to the particle phase while the simulation is running .....

Figure 9 - Plot of particle motion due to Brownian motion over 10,000,000 time steps.

Figure 10 - Plot of the root-mean square values of the particle distance travelled by diffusive motion. 
Figure 11 - Particle plots of the velocity profile for the Bruus and Pozrikidis solutions. a,b) The respective Bruus and Pozrikidis velocity results for the data in Table 8. c,d) Three dimensional velocity profile of 1000 random points in the respective Bruus and Pozrikidis equations, average particle velocities are shown above.

Figure 12 - Separation results from flow rate of $5 \mathrm{e}-6 \mathrm{~L} / \mathrm{min}$ at variable voltages ranging from $0-50$ volts. The voltage was applied over a 200-micron channel to generate the electric field. a) Percent yield of large particles (radius 2.6 microns) b) Percent yield of small particles (27nanometers) c) Purity of large particles d) Purity of small particles.

Figure 13 - Separation results from flow rate of $10 \mathrm{e}-6 \mathrm{~L} / \mathrm{min}$ at variable voltages ranging from $0-50$ volts. The voltage was applied over a 200 -micron channel to generate the electric field. a) Percent yield of large particles (radius 2.6 microns) b) Percent yield of small particles (27nanometers) c) Purity of large particles d) Purity of small particles

Figure 14 - Separation results from flow rate of $15 \mathrm{e}-6 \mathrm{~L} / \mathrm{min}$ at variable voltages ranging from 0-50 volts. The voltage was applied over a 200-micron channel to generate the electric field. a) Percent yield of large particles (radius 2.6 microns) b) Percent yield of small particles (27nanometers) c) Purity of large particles d) Purity of small particles.

Figure 15 - The separation results from a simulation with extended channel length, L. The flow rate for this fractionation was 10 microliters/minute at a variable voltage ranging from 10-30. a) Percent yield of large particles (radius 2.6 microns) b) Percent yield of small particles (27nanometers) c) Purity of large particles d) Purity of small particles 


\section{Introduction}

The goals of this thesis are:

- To develop a computational model of a microfluidic separation process that can determine the feasibility of separating like-charged particles in a continuous flow environment.

- The model must capture enough of the physics behind separation processes to produce reasonable results.

- To conduct computational experiments to evaluate the model and to investigate the separation of like-charged particles

- This simulation was produced with Matlab® software; as this package is optimized for the use of matrices the code was to be developed in a vectorized form.

- A limitation of many colloidal simulations is the existence of particle-particle overlap; to resolve this a goal of this thesis was to mitigate this overlap condition and, by doing so improve the accuracy of the model.

In order to meet these goals the following questions must be answered.

1. What are the physics that drive a separation process?

2. How is the separation of like charged particles different from other fractionations, and how can these differences be utilized?

3. What other computational models of fluid-particle systems exist and what can they contribute to this project?

4. How do computational systems efficiently perform collision detection and response on large numbers of objects? 


\subsection{Motivation}

In the fields of diagnostics, e.g., therapeutics, cellular biology, the ability to separate and isolate specific particle types in a microfluidic system is essential. The medical field has seen a shift toward minimally invasive treatments and point-of-care diagnostics, which require new technologies and methods to facilitate such a transformation. A branch of research and development that has grown to meet this need is microfluidics $[1,2]$. These devices are capable of performing processes and assays previously limited to a laboratory environment. By utilizing these devices and improving their capabilities point-of-care options can increase and improved diagnostic systems can be developed.

This paper will focus on the separation of like-charged particles and the optimization of processes using a computational simulation.

\subsection{Clinical Diagnostics}

Current diagnostics rely on laboratory based sample preparation and processing; while this method is effective it is tedious and expensive. A typical lab environment employs several technicians to maintain and operate bench-top equipment. In diagnostics sample preparation is a crucial step leading to analysis; it is used to purify the sample and bring the concentration of target species to a level that can be detected. Some of the techniques utilized in clinical laboratories are: centrifugation, filtration, distillation, dilution, target amplification, and extraction. An example of a processing procedure is the preparation of a blood sample in a clinical laboratory. The typical processing of a blood sample in a clinical diagnostic lab begins with the centrifugation of the sample tube containing the blood of the patient. This separates the blood into three layers: erythrocytes, plasma, platelets and leukocytes. Following this fractionation the samples are further divided into several aliquots for testing. Each of these 
samples is used for different evaluations. The samples may then be mixed with reagents or markers before analysis by flow cytometry or ELISA.

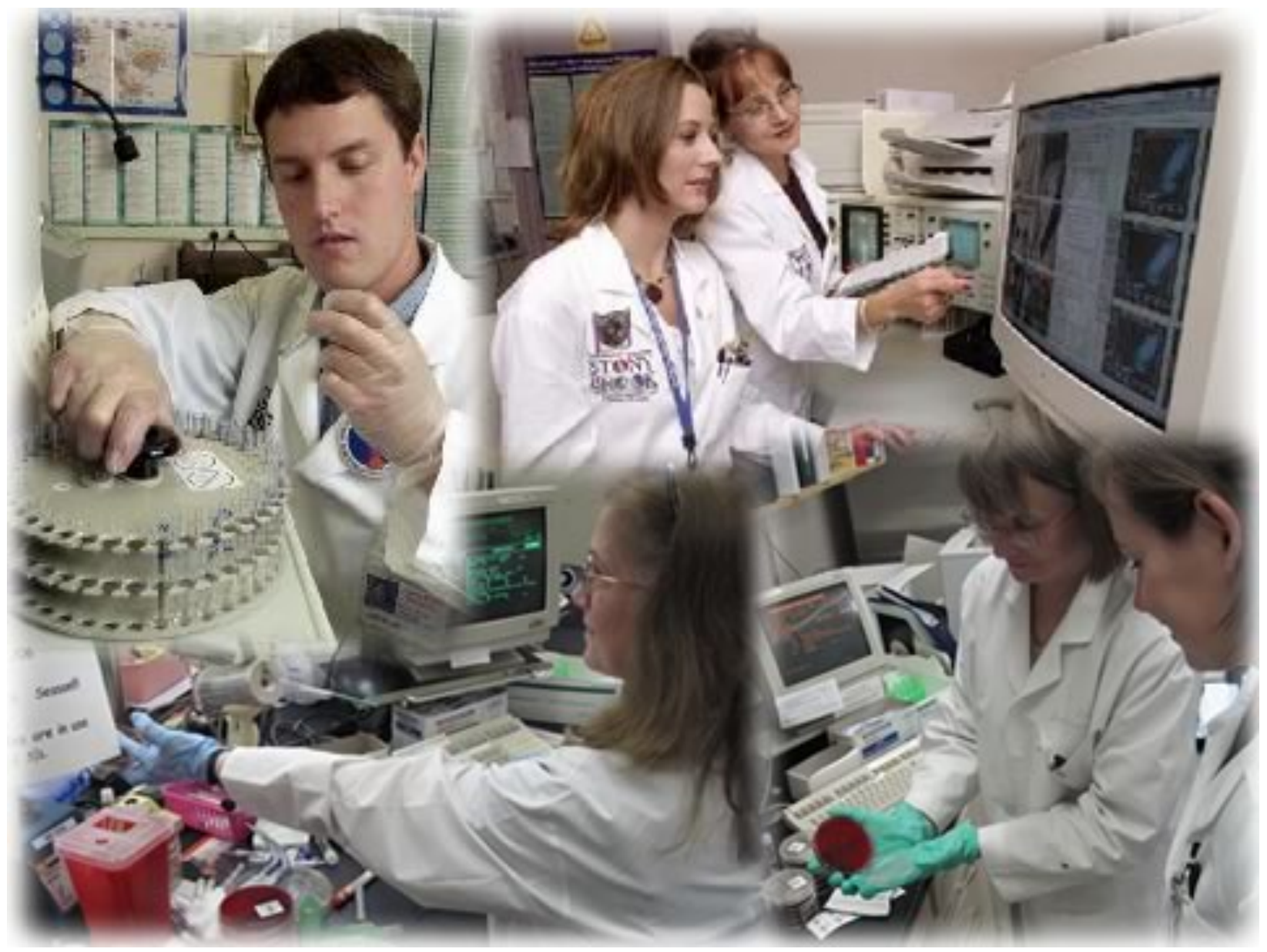

Figure 1 - Compilation of images from a clinical diagnostic laboratory. The upper left displays a technician loading a centrifuge with vials of biologic samples. The upper right shows two technicians viewing results from a test. In the bottom right, two individuals examine a culture dish. The bottom left shows a technician working with sharps.

\subsection{Microfluidics}

Lab-on-a-chip technologies have developed over the past 2 decades to replace or supplement laboratory functions. These microfluidic devices are small compared to the bench-top equipment typically utilized in clinical settings however they have the potential to improve the sample preparation process [3]. These devices may be capable of performing one or several laboratory functions, reducing the need for larger equipment. Initially microfluidic devices were primarily 
used in the analysis of samples while pretreatment and processing took place outside the system [4]. This focus was driven by the advantages of microsystems over conventional systems, such as capillary electrophoresis compared to gel electrophoresis. The developmental focus has since shifted to move the processing and pre-treatment onto chips as well as analytic techniques.

Figure 2 displays the flow of sample from the raw state through processing, analysis, and interpretation. By combining these steps on a chip the lab space and time for analysis can be reduced as well as the required sample volume. Due to the many techniques used in clinical laboratories a lab-on-a-chip device may need to employ several sequential steps and methods to prepare and process a sample [5].

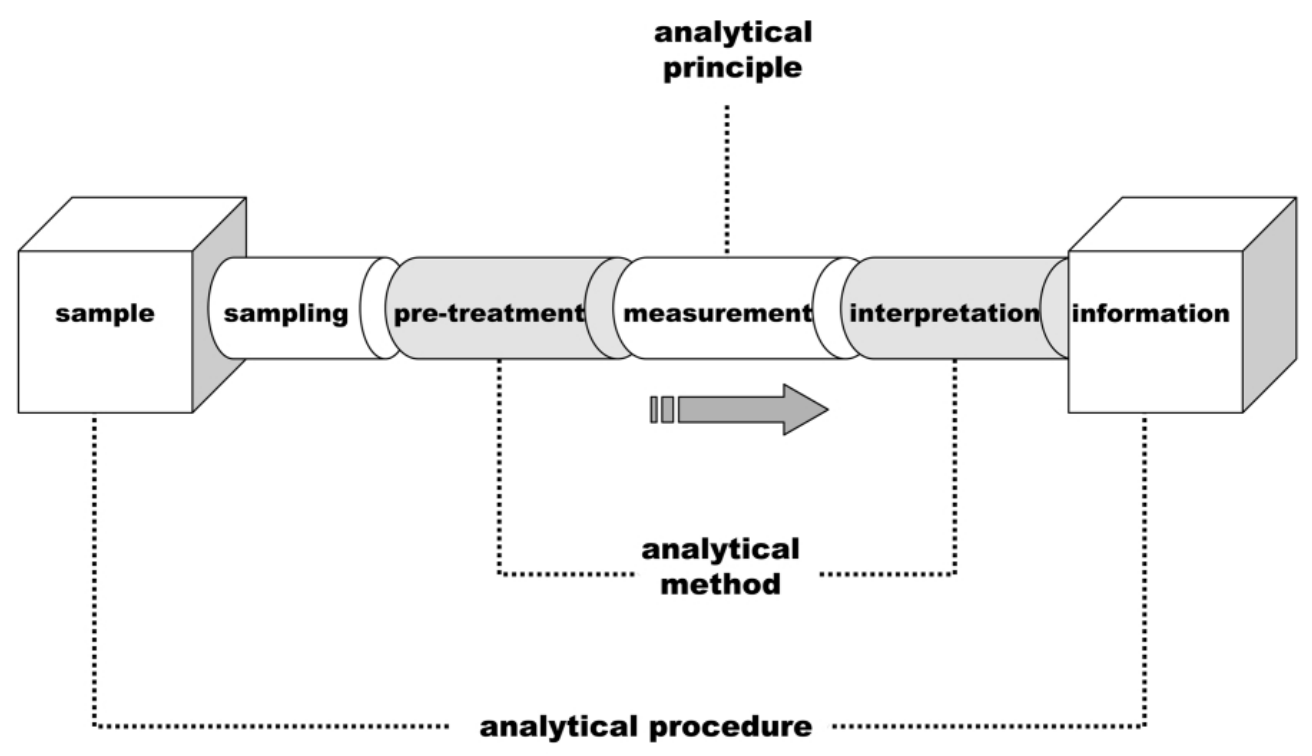

Figure 2 - Diagram of the modular analytic process. The boxes on the ends correspond to the generic input sample and the information gained from it. The four cylindrical steps in the middle follow the sample from the raw state through pre-treatment and processing, after which it is analyzed and interpreted.

\subsection{Sample Preparation}

After a sample is collected from a patient it undergoes a series of preparatory processes before analysis. The first of these steps is raw sample introduction; this involves taking the sample from the patient, without any modifications or processing, and introducing it into the system.

Next the sample is prepared for further processing by pre-conditioning; this may include but is 
not limited to altering the $\mathrm{pH}$, ion concentrations and viscosity to change separation properties. Following pre-conditioning, a separation process is used to divide the sample into species based on size; an example could be fractioning cells from proteins. Having narrowed the species in the sample it is further separated to isolate a specific colloid or group of species, such as a particular protein. Once the target particle is isolated it undergoes pre-concentration to enable detection by the analytic system.

\subsection{Separations}

The separation process selected for a given diagnostic sample is based on the properties of the species contained in the sample. As these properties determine the efficacy of a process, it is important to understand the sample type and the particle species typically found within it. Separation techniques utilize the unique properties of the target species, referred to as handles, that an enable preferential separation of the target species from the bulk material. These handles include physical, chemical, morphologic, and electrical characteristics of the particles [6].

Physical properties refer to the size, shape and mechanical properties of the species. Many separation methods utilize size as the primary separation handle, including hydrodynamic chromatography and filtration. Another property that impacts separations in a similar manner to physical properties is morphology. Although mammalian cells have similar morphological properties there are significant differences compared to proteins, viruses and bacteria.

Electrical charge is another distinguishing "handle" utilized in separations, most commonly in electrophoresis. By manipulating a charged particle with an external electric field the target species can be propelled along the direction of the applied field, independent of the suspending medium. In the case of a flow environment, electrical charge may be manipulated to redirect the motion of a colloid from its original streamline in the fluid [6,7]. Electrical charge also exerts 
an influence on inter-particle interactions, driving an attractive or repulsive force between particles. These potentials result from dipole-dipole interactions or induced dipoles. The interacting dipoles may be permanent dipoles or could be induced dipoles, created by the application of an external electric field. The affinity of particles has a similar effect as the interparticle interactions, resulting in species migration without external interaction. The polarizability of species can be exploited using electrical influences as well; polar and non-polar particles can be oriented or directed with electric fields through dielectrophoresis.

Dielectrophoresis utilizes a non-uniform field to polarize both the particles and the solution to generate particle motion, where the magnitude is related to the colloidal polarizability relative to the solution [3].

Chemical properties that impact and can be utilized in separations include ligand-receptor affinity. Due to the attraction and interaction of these colloids, inter-particle binding can occur during separations. This ligand binding can be implemented in a separation as a technique to isolate specific cells. By tethering ligands to the channel walls, cells with the receptors can bind and, similar to leukocyte rolling and tethering, slowly roll through the channel [8]. After the tethering and rolling the cells are re-suspended in the fluid and allowed to elute from the microfluidic system. This type of separation is capable of producing high concentrations of the target cells from the injected samples [9].

During the processing of biological samples certain separations may be limited by the properties of the target species. An example of this is the separation of Monocytes from Platelets. While these particles differ in size they have a similar surface charge, making electric field driven separations difficult or ineffectual, especially for high volume samples. 
Particle separations can be categorized as either batch flow or continuous flow processes. In a batch-flow system a finite bolus of the sample is injected into the device. This sample then undergoes processing that segregates the agglomeration of species into similar groups. Batch flow systems are limited to relatively small samples, which translates into fewer particles in the separation. Examples of batch flow systems are chromatography, electrophoresis, centrifugation and filtration. This type of processing yields high purity but due to the limitations on initial sample size the end product is small compared to other techniques.

A continuous flow system utilizes a continuous injection of sample. The species to be manipulated are separated during their flow through the system and, depending on the separation method, are collected at varying points along the way through the system or at different times or locations at the device outlet. The individual species are fractionated using a variety of techniques, including pinched flow fractionation, hydrodynamic filtration, and free-flow electrophoresis. Continuous flow separation systems can be efficient, re-useable and capable of handling large sample volumes.

Having touched on the fundamental characteristics of separation methods, it is necessary to understand the metrics by which a fractionation process is evaluated. Separations are evaluated based on yield and purity. Yield is the percentage of target isolated and collected relative to the total amount of target in the original sample, as shown in the expression

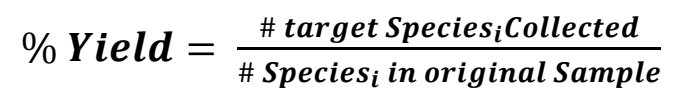

where the number of particles collected refers to those captured for analysis, and the number in the original sample is the number of colloids injected. The subscript $\mathrm{i}$ identifies the species type, 
determining the yield of each species injected into the separation channel. Purity is the percentage of a species collected relative to other species in the output.

$$
\% \text { Purity }=\frac{\# \text { target } \text { Species }_{i} \text { Collected }}{\text { Total } \# \text { of species Collected }}
$$

where the number of a specific species collected is related to the total number of particles collected. A high purity means that the collected sample is mainly composed of the target species. These metrics are useful in the evaluation of separation efficiency; by maximizing these values within a process smaller samples can be utilized to obtain the necessary final concentrations of particles.

\subsubsection{Continuous Flow Separations}

The use of microfluidic devices has typically been limited to batch flow processes however developments have increased the utility and prevalence of continuous flow separations. These types of separators have many advantages; first and foremost the continuous nature of the process accommodates large sample volumes and maintains separation efficiencies comparable to batch separators. Like batch separators, continuous separators can leverage the same intrinsic properties of target species to fraction the sample into components [10]. An illustration depicting the differences between batch separations and continuous flow processes is shown below. 

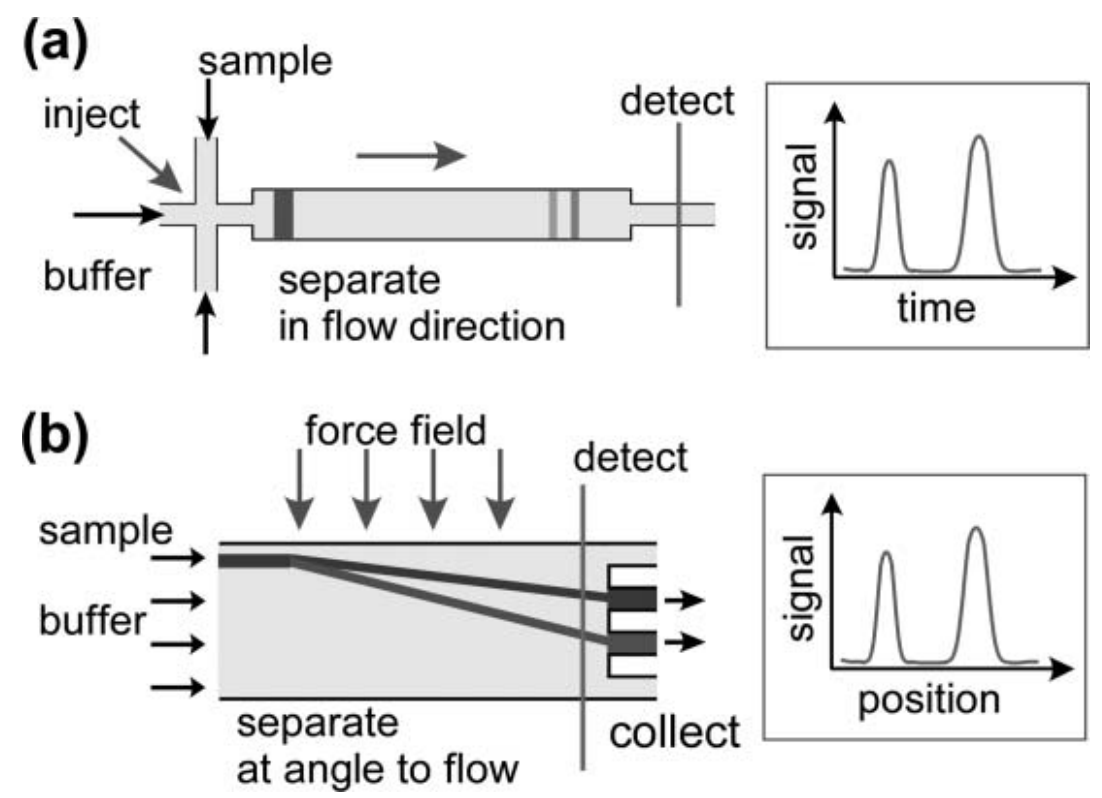

Figure 3 - Comparison of batch flow separations with continuous flow systems. a) Drawing of a batch flow fractionation device and the elution profile for separated species. b) Diagram of a continuous flow system and the elution profile of its separated species.

Many methods of continuous flow fractionation have been developed and while they vary in mechanisms of action they all share a few common characteristics. A sample is driven, typically with a buffer fluid, through a channel where its flow is deflected or altered by a force or obstacle (Figure 3b). The combined influences on particle motion facilitate collection as the species elute from the end of the channel or at points along the channel, at outlets. Variations in separation techniques require different system configurations to optimize the process.

\subsubsection{Hydrodynamic Chromatography}

Hydrodynamic chromatography is a separation phenomena similar to size exclusion chromatography. The driving force behind this separation process is a pressure-driven solvent flow. The diffusion of solute particles throughout the channel results in sampling a range of fluid velocities from the parabolic flow profile; as these particles traverse the channel they follow the fluid streamlines. Particle diffusion is based on the Stokes-Einstein diffusivity equation, shown below. Due to their size, large particles are limited to the faster flow regions of the 
channel whereas the smaller particles access the slower flow regions near the channel walls

(Figure 4). This results in a higher velocity for the large particles compared to the small particles.

When the channel is sufficiently long the particles can be separated by size, as the larger

particles will reach the end before the smaller particles [11].

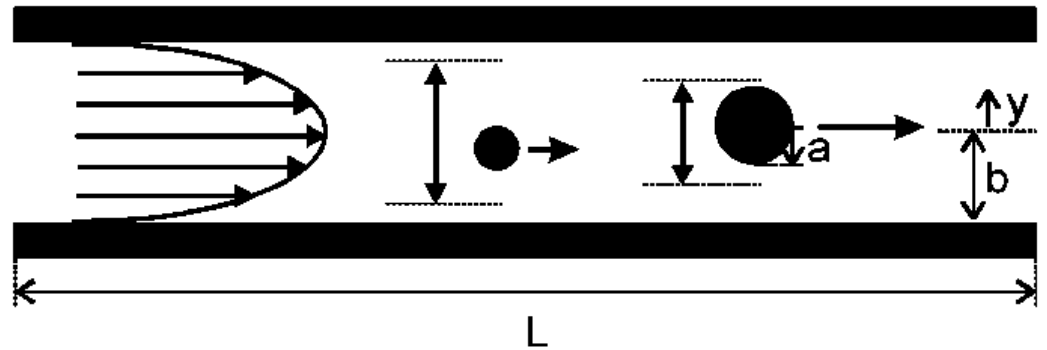

Figure 4 - Conceptual drawing of Hydrodynamic Chromatography. The large particle is limited to the high flow region of the channel while the small particle is able to acces slower flow areas. The diffusion of species results in a higher large particle average velocity than the small particles, leading to elution of the large particles first.

$$
D=\frac{k T}{6 \pi \eta a}
$$

The Stokes Einstein diffusivity, D, determines the diffusivity of a particle from its radius, a, the fluid viscosity, $\eta$, and the fluid temperature, $T$. The value $\mathrm{k}$ is the Boltzmann constant, $1.3802 \mathrm{e}^{-23}$ $\mathrm{J} / \mathrm{K}$.

Hydrodynamic chromatography is an effective separation process however it is limited to narrow conduits $(\leq 1 \mu \mathrm{m})$ with the large particle diameters ranging from 0.002 to 0.2 of the conduit size. This characteristic restricts the process from higher volume separations [12].

\subsubsection{Electrophoresis}

Electrophoresis is a separation technique that utilizes an electric field in conjunction with the charges of the target species. This process is based on migration of solutes toward the electrode of the opposite charge. As this technique is based on the Electrokinetic properties of the colloids the separation depends on the distribution of charges. 
A diffuse layer screens the species according to double layer theory. This screening layer is composed of oppositely charged ions and has magnitude equal to that of the solute.

Electrophoretic systems are typically configured to operate in one or two dimensions. Onedimensional electrophoresis most often implemented for the separations of proteins or nucleic acids while two-dimensional systems are useful for more complex compounds. A common application of electrophoresis utilizes gels as the solvent or medium that the species travel through. In the realm of microfluidics and separations electrophoresis may be employed to drive charged species through a fluid, resulting in a separation gradient by charge. The species are then collected as they reach the end of the system.

In the field of microfluidic separations capillary electrophoresis has become a prevalent technology both in research and clinical settings. The high separation efficiencies of this technique have driven the development of this method, which has demonstrated success in the clinical separations [13].

Free flow electrophoresis (FFE) is a development on gel and batch flow electrophoretic techniques. This method utilizes a laminar fluid flow in conjunction with an applied electric field to influence the particle paths across the channel [14]. By manipulating differences in the electrophoretic mobilities of the species they can be directed by the electric field. The electrophoretic mobility, $\mu_{\mathrm{e}}$, of a colloid is determined by its electrical charge and relationship to the surrounding fluid, as shown by

$$
\mu_{e}=\frac{\varepsilon_{r} \varepsilon_{0} \zeta}{\eta}
$$

where $\varepsilon_{\mathrm{r}}$ is the reference permittivity of the solvent, $\varepsilon_{0}$ is the dielectric permittivity in a vacuum, $8.8542 \mathrm{e}^{-12} \mathrm{C}^{2} /(\mathrm{J} * \mathrm{~m})$. The other elements of this equation are: the zeta potential, or electrokinetic 
potential, $\zeta$, which correlates the particle charge to the electric double layer of the fluid, and the fluid viscosity, $\eta$. Having determined the electrophoretic mobility of a colloidal particle in solution, its motion from an applied electric field is given by

$$
\mu_{e}=\frac{v}{E}
$$

where $\mathrm{v}$ is the particle velocity, and $\mathrm{E}$ is the magnitude of the electric field.

The typical configuration of this system has the electric field oriented perpendicular, across the flow of fluid. The sample is injected at one of the channel walls and is carried by a carrier fluid continually flowing through the system. The electric field deflects the particles into streams according to their mobility; at the end of the channel the fractionated species are collected at different locations.

The efficiency of free flow electrophoresis in a given system depends on the parameters used in the setup, most importantly fluid flow rate and magnitude of the electric field [15]. It should be noted additionally that the channel dimensions impact the separation properties; however, dimensions are less easily adapted to optimize fractionation efficiency than flow rate and electric field strength.

\subsubsection{Electric Field-Flow Fractionation}

Field flow fractionation (FFF) is a process capable of separating colloids and particles based on multiple properties. It utilizes a constantly flowing fluid buffer with an additional force applied perpendicular to the flow. This technique is similar to chromatography-based techniques in that a single bolus of the sample is injected and the fractionated species are collected as they are eluted, as in a batch flow system. Field flow fractionation differs in its manipulation of colloidal 
properties, specifically diffusion. As the external force propels the particles to a wall of the channel the diffusive motion of the species lift the particle off the surface. As the diffusive motion of species is determined by their properties the particles will sample different flow velocities, similar to hydrodynamic chromatography. Due to the laminar parabolic nature of the fluid flow in FFF the particles closest to the wall will travel at a lower velocity than particles diffusing closer to the midline of the channel. The particles traveling at higher velocity reach the end of the channel before the others, allowing collection of individual species [16].

Three main modes of field flow fractionation exist: normal mode, steric mode, and hyperlayer mode [17]. The normal mode utilizes an applied force to shift the particles to a channel wall however the force is sufficiently low to allow diffusive motion of the colloids, which allows the elevated diffusive motion observed in small particles $(<1 \mu \mathrm{m})$ from Brownian motion to move the species from the channel wall to higher velocity regions of flow. In this modality the smaller species elute before larger particles however due to the size limitation many biologic species cannot be separated in this manner. The steric mode of separation is utilized for particles larger than $1 \mu \mathrm{m}$, where diffusive motion plays a subdued role. In this technique the particles are pinned to the wall by an applied force sufficient and, due to their size the larger particles access a higher velocity stream than the smaller particles. This results in the elution of large particles first, followed by small. The hyperlayer mode, also referred to as the lift mode, utilizes a high velocity fluid flow to generate hydrodynamic lift forces that drive the species toward the channel midline (Figure 5). This results in elution in the same order as in the steric mode; large followed by small. Particles from a wide range of sizes, $1 \mathrm{~nm}$ to $100 \mu \mathrm{m}$ can be separated with high resolution using these techniques [18]. 


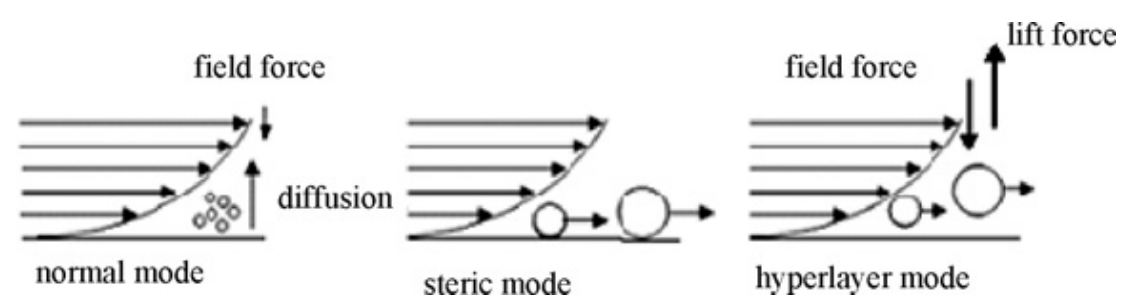

Figure 5 - Conceptual drawing of the three modes of electric Field Flow Fractionation. Left) The normal mode, a low force and diffusive motion of particles drive the separation. Middle) Steric mode, a high force and hydrodynamic effects drive the fractionation. Right) Hyperlayer mode, high flow rate and force lift and separate the particles by hydrodynamic forces

FFF utilizes several mechanisms to drive the force perpendicular to the flow including thermal gradients, cross flow of fluids, magnetism and electric fields. Electric field flow fractionation (EFFF) in particular has been cited for its utility in biological separations [19]. Its capabilities have been demonstrated on cells, colloids and organelles, differentiating it from the limitations of electrophoretic separations. Like free flow electrophoresis this technique uses the electric mobility to propel the particles with an electric field [20]. This exerted field drives the species to the wall where one of the free flow fractionation modes separates them. The elution of species from an EFFF system resembles a batch flow system; the separate species reach the end of the channel at different times. Even though this differs from continuous flow techniques electric field flow fractionation has the ability to separate species at higher resolution than other methods.

\subsection{Separation of like charged species}

In a solution of like charged particles available separation techniques are limited, specifically those utilizing charge as the separating handle like electrophoresis. Charge based techniques may be utilized in batch separation systems however in a continuous flow environment the species become may become congested if the electrophoretic mobilities of the species are similar, decreasing the efficiency of the system. In addition to the implementation of an electric field, the colloidal interactions can be utilized to aid in the separation. Electrostatic forces between species play a key role in the physico-chemical properties of the solution [21]. Derjaguin, 
Laundau, Verwey, and Overbeek (DLVO) theorized that these interactions consist of repulsive and attractive forces, based on the distance between species. The attractive van der Waals force is based on the Hamaker constant and the separation between the particles [22]. The attractive force between two plates is given in the equation:

$$
U_{V D W}=-\frac{A_{H}}{12 \pi h^{2}}
$$

where $h$ is the separation between the plates and $A_{H}$ is the Hamaker constant. The repulsive component of DLVO interactions is based on a combination of fluid and particle properties. The resulting potential serves as a repulsive force between particles as a function of separation distance, given by:

$$
U_{E D L}=32 \varepsilon_{0} \varepsilon_{r} \kappa \gamma_{1} \gamma_{2}\left(\frac{k T}{z e}\right)^{2} \exp (-\kappa h)
$$

where $\varepsilon$ is the electric permittivity, $\gamma$ represents the surface charge of the particles, and $x$ is the inverse Debye length. The values k, T, z, e, and h are the Boltzman constant, solution temperature, valence of the electrolyte solution, electron charge, and separation distance between interacting surfaces, respectively. The combination of these potentials yields the attractive and repulsive phenomena cited by DLVO.

In 2007 a research team at Beilfeld University in Germany investigated the use of a pulsed electric field to separate like charged particles. In their research they found that such species could be effectively divided into individual types. They noted that when the pulsed field was applied the particles traveled in opposite directions. Although the mechanism behind this phenomenon is not entirely understood they believe that it is related to thermal noise within the system. Research such as this suggests that novel uses of electric field mediated separations may be increasingly useful in the separation of like-charged particles [23]. 


\subsection{Computational Modeling of Separations}

Due to the complexity of separation processes and the numerous variables that contribute, computational models are useful to set up and validate experiments and procedures before performing them in a laboratory setting. In order to ensure the model accurately represents the process it is necessary to capture a sufficient amount of the system's physics. This includes the properties of the fluid and particles, as well as the system dimensions, flow characteristics, and external influences contributing to the separation. By assembling these parameters in an algorithm a separation can be tested and calibrated to achieve optimal fractionation of species. In addition to testing and validation, computational models can augment the design of microfluidic devices by running experiments prior to manufacture. The application of modeling to design processes has the potential to greatly improve development of quality devices.

Several approaches exist for the computational modeling of complex particle systems. Finite element analysis software, such as COMSOL $®$ simulates the entire system as a mesh, where forces and changes impact all other aspects of the system through fluid-particle and particleparticle coupling. Additional numerical methods have been developed to simulate these solventsolute interactions using a variety of programing languages and development platforms.

\subsubsection{Multi Particle Interactions}

In order to accurately model a dynamic fluid-colloid system the interactions between particles must be accounted for. During the transport of solutes the fluid and inter-particle forces will contribute to the separation [24]. These forces may result from particle collisions with other particles or system boundaries, as well as electrostatic and Van der Waals forces. To account for these contributions to colloidal movement a solution technique must be selected. Several theoretical and computational models for these interactions exist, a few of which will be briefly described below. 


\subsubsection{Molecular Dynamics}

In order to account for the interactions of particle and fluids the system can be simulated as molecules and particles in a molecular dynamics (MD) model. This method simulates each molecule in the system and utilizes the interaction potentials between molecules to determine the inter-particle forces [25]. Although this type of simulation has a high degree of accuracy the model is computationally expensive due to the number of interacting particles.

\subsubsection{Brownian Dynamics}

The diffusion of particles from Brownian motion describes the transient distribution of colloids throughout a volume. By quantifying this particle travel in a computational simulation, complex systems can be modeled and better understood. Brownian dynamics primarily analyses the propagation of particle trajectories through a solution by diffusive motion. These particles are typically assumed to be non-interacting; the particle paths are independent, modeling the system as isolated colloids [26]. Particle motion is determined by the Stokes-Einstein diffusion coefficient and the net force applied to the particle. During the calculation, the diffusion coefficient is multiplied by a random value to maintain a linear variance [27]. The particles are initially inertialess during each time step, eliminating momentum from the calculation. During the evaluation of Brownian dynamics a sufficiently small time step is selected to minimize the particle motion during any one increment of travel.

The solution to Brownian dynamics is based on the Langevin equation for motion,

$$
m \ddot{r}=F_{\text {force }}+F_{\text {Brownian }}
$$

where $\mathrm{m}$ is the particle mass, $\ddot{r}$ is total acceleration, and $\mathrm{F}$ represents net Brownian force and other forces acting upon the particle. By assuming that the time-averaged Brownian force equals 
zero and integrating this equation across the time steps of the simulation the particle motion is found [28]. This particle motion, $\Delta \mathrm{r}$, calculated from this model is given by

$$
\Delta r=\frac{D F}{k T} \Delta t+S=\frac{F}{6 \pi \mu a} \Delta t+S
$$

where $\mathrm{D}$ is the Stokes-Einstein diffusivity, $\mathrm{F}$ is the net force applied to the particle, $\mathrm{k}$ is the Boltzmann constant, $\mathrm{T}$ is the solution temperature, and $\mathrm{S}$ is the stochastic term for Brownian motion [29]. In the right portion of the equation, D/kT has been replaced by the term for Stokes Drag, where $\mu$ is the fluid viscosity and $a$ is the particle radius. The stochastic component of Brownian motion is given by

$$
\left\langle S^{2}\right\rangle=2 D \Delta t
$$

where $\mathrm{D}$ is the Stokes-Einstein diffusivity and $\Delta \mathrm{t}$ is the duration of the time step, as given by the Ermak-McCammon equation [30]. The time steps for these simulations are small, typically on the order of several thousandth of the total simulation time.

\subsubsection{Lattice Models}

Another model utilized in the simulation of fluids and particles is the lattice Boltzmann model.

This utilizes a fixed lattice that fluid particles travel along. Colloidal particles diffuse and flow freely through the volume, impacted by interplay with fluid particles. Particle interactions occur when multiple particles arrive at the same point. The resulting collision dynamics are typically elastic to maintain momentum but may account for additional inter-particle forces. A limiting factor of lattice models is Galilean invariance as the particle motion is fixed to a grid.

\subsubsection{Direct Simulation of Monte Carlo}

Direct Simulation Monte Carlo (DSMC) is a fluid simulation that models the system as particles. These particles represent regions of fluid and are assigned position and velocity values that 
iterate through a series of time steps. In this model each time step consists of a streaming phase and a collision phase. The system is divided into a grid; the particles within any given grid box can collide [31]. Collisions are between pairs of particles and are determined using a probability model. DSMC simulations represent the bulk fluid body; however, they are computationally expensive. Additionally, the model is targeted towards fluid simulations rather than fluidparticle interactions.

\subsubsection{Stochastic Rotation Dynamics}

Stochastic Rotation Dynamics (SRD), also known as multi-particle collision dynamics, is a relatively new model of particle dynamics that accurately models the solute-solvent interactions. This technique captures both the fluid influences on the particle as well as the particle influences on the fluid. Unlike the Lattice-Boltzmann methods this simulation allows for free flowing solute and fluid independent of a lattice or node based system.

The fluid is modeled as points or particles of infinitely small volume. This fluid is then subjected to two phases: a streaming step where the particle velocities are determined and applied, and a multi-particle collision dynamics (MPC) step where the fluid-fluid interactions are taken into account. In this model the MPC phase is determined using a lattice to divide the volume into Wigner-Seitz cells. The particles within each grid region are rotated using a random rotation matrix, with the rotation of each cell independently generated [32].

The solute components are similarly modeled as particles with two phases of motion; however these particles have a finite volume and mass [33]. These particles are subjected to the random rotation of the solvent particles but have an additional solute-solute interaction through a Lennard-Jones potential. 
In order to capture the dynamic interactions of the fluid and particles a random shift is applied to the Wigner-Seitz cells during each time step to maintain Galilean invariance [34]. This model is highly accurate at capturing the multi-particle dynamics of colloidal systems however is computationally expensive due to the particle representation of the fluid. Stochastic rotation dynamics have been evaluated and with complex shapes such as DNA in microfluidic separations [35]. In this simulation the computational results were confirmed by experimental data, supporting the accuracy of this model.

\subsection{Current Separation Models}

Several models have been developed and validated for separation processes. Free flow electrophoresis is a commonly utilized separation technique and the physics governing it are documented. A model for protein separation by FFE was developed under the hypothesis that such a simulation could aid in the selection of system geometries and simulation parameters [36]. This model was based on the mass-transfer flux of the species and the contribution of the electric field through the electrophoretic mobilities of the particles. Additionally a hydrodynamic component was included to account for the fluid-particle interactions. This model succeeded in simulating a protein separation however had limited experimental data to validate the results. Although limited in that sense, the model supported the hypothesis that computational modeling could aid in the determination and optimization of separation parameters.

Field flow fractionation has been modeled to improve the efficiency and efficacy of separation processes. A collaboration of researchers as Baxter Healthcare, Proctor and Gamble, and CFD Research developed a simulation of dielectrophoretic FFF systems [37]. Due to the complexity of such a separation a simulation can aid in the understanding of the forces acting on the particles. The goal of this model was to assist in the selection of geometry and simulation parameters in 
order to improve performance. The separation in this model is driven by Navier-Stokes fluid flow in conjunction with the dielectrophoretic force. The algorithm used assumes small particles in a point-particle model. The iterative portion of the simulation utilized a resolved-particle method, determining and applying flow interactions and electric field contributions to the particles in each time step. This model was not directly compared with experimental data however the distribution of species throughout the system agrees with the theoretical results.

The use of computational simulations of separations potentially holds a key role in the development of improved processes. By using models to gain a better understanding of these processes efficiency can be increased. Additionally these models can be used in the design of separation systems, validating and optimizing the process computationally.

\subsection{Project Overview}

Computational simulations of bioseparation processes are typically performed on high power computers. Although models exist for personal computers they are often tedious or limited by memory and processing capacity. Certain software platforms exist with the capability to handle complex simulations and calculations; one such program is Matlab®. It has been utilized for modeling fluid systems, often in conjunction with another program such as COMSOL however if optimized properly Matlab® can handle separation simulations. By capturing enough of the physics in a separation process within the simulation accurate results can be produced, leading to more efficient and optimized systems. Such a simulation could assist in the calibration of existing separation systems as well as in the design of new systems.

The simulation developed in this thesis models the separation of large and small particles in a microfluidic system and aims to answer the question: Can like-charged solutes be separated with high yield and purity in a continuous flow environment? 
The goals of this project include (1) writing a simulation that captures a sufficient amount of the physics to respond to the hypothesis, (2) conduct computational experiments with the model to determine validity, (3) utilize vectorized code within Matlab®, (4) design the simulation such that particle-particle overlap is eliminated. By accomplishing these goals a simulation will be produced capable of representing a separation process on a personal computer.

In the next sections, the following topics will be discussed.

- Development of the computational model

- Testing and Validation

- Results

- Conclusion

\section{Model Development}

This section discusses the development of the computational model. MATLAB was selected as the software package to handle the simulation. This program has a built-in library of functions and capabilities that aid in the construction of the code. The purpose of this model was to simulate the separation of like-charged particles in a continuous flow environment. Research suggested two techniques that were identified as potentially advantageous in this type of simulation: electric field flow fractionation and free flow electrophoresis. Both of these separation methods utilize the electric charge of the particles and have a similar apparatus, the primary difference being the manner of particle elution from the system. To review, these systems utilize a continuously flowing buffer fluid through the channel; the sample is injected at the channel entry near a wall and an electric field either deflects the particle paths or pushes them to channel wall. As the mechanics and physics behind both of these techniques are similar it 
seemed reasonable to design the simulation such that it was capable of modeling either technique with minimal alteration. The outputs of this simulation are an animated visualization of the separation process (the particles traveling through the channel), the yield and the purity of species collected by the system.

\subsection{Particle Modeling}

Having determined the type of simulation to be modeled, the algorithm and technique to represent the process were selected. This model was influenced by Brownian dynamics, molecular dynamics, direct simulation Monte Carlo, and stochastic rotation dynamics. Initially simulations directly based off of DSMC and SRD were investigated, but were eliminated due to the computing power required for these models. A Brownian dynamics type approach was selected for the simulation; however, an adapted model was developed. The model utilized aspects of DSMC and SRD, particularly the two-phase particle motion. In this model, and unlike the point-particle method used in molecular dynamics, the particles have a volume that utilized in the calculation of inter-particle dynamics.

Brownian Dynamics formed the foundation of this simulation; however, it was inspired by several other methods. The three models contributing to this project, MD, DSMC, and SRD all modeled the fluid as particles traversing the channel; however, in this Brownian dynamics type model, the solvent is treated as a stochastic kick. This models the fluid influence on the particles as a statistical kick by Gaussian statistics. By representing the fluid in this manner rather than by solvent particles, such as in MD, DSMC, and SRD, computational time is saved. Regardless of the type of model used, the type of flow plays a key role in the separation dynamics. For microfluidic devices the dimensions support the assumption that flow through a channel is laminar and parabolic. 
By combining the fluid and particle components of this system using super-position the model construction can begin. As stated above this simulation was inspired by and implemented the two-phase particle motion utilized in other models [38]. These two phases are Particle Motion and Particle Interaction; both phases occur within each time step of the simulation. The particle motion phase includes of the application of forces and resulting displacements to the particle from sources excluding other particles. These include the application of fluid velocity, diffusion, and external forces like electric fields. The particle interaction phase accounts for the interparticle reactions: repulsion energy and collisions.

Due to the iterative nature of this simulation, the resolution of the time steps plays an important role. The total simulation time is determined by the residence time, found by dividing the total channel volume by the volumetric flow rate. The simulation time is divided by the number of steps, input by the user, to determine the length of the time steps.

\subsection{Particle Motion}

\subsubsection{Diffusion}

Particles in a fluid diffuse freely, following random trajectories due to the influence of Brownian particles. This phenomenon is present in all separations. One such method that directly utilizes diffusion is the normal mode of field-flow fractionation, where the diffusive motion of small particles is significantly greater than that of large particles, resulting in separation of species by diffusivity. Stokes and Einstein characterized and described the diffusivity of particles (Eqn. 3) utilizing the fluid properties (temperature and viscosity) and the particle size to determine the motion of the particle from diffusion [39]. The colloidal motion resulting from this equation supports the elevated diffusive potentials that small particles have over large species. 
In order to simulate this computationally a random walk is calculated for the solute particles; this results in motion along a single vector. This displacement, modeled as a random kick, represents the self-diffusion of a particle from the Brownian forces upon it. The equation below shows the calculation of the kick magnitude from the Stokes Einstein diffusivity, $D$, given in Eqn. 3 .

$$
k i c k=\sqrt{2 * D \Delta t}
$$

The value generated from this is then multiplied by a random value to simulate the Brownian motion of the fluid. This random kick is generated in each of the three Cartesian directions for each particle; by combining these, the three-dimensional diffusive motion is determined. During each time step this kick is randomly generated for all particles in the simulation, contributing to the total motion of colloids throughout the model. This random kick is traditionally the foundation of Brownian dynamics.

\subsubsection{Electric Field Contribution}

The primary force driving the separation of injected particles is the applied electric field. In literature, free flow electrophoresis and electric field flow fractionation been demonstrated in several techniques and configurations to fractionate species based on charge and size. In this model the application of an electric field is intended to deflect and move particles to a different location or path through the channel. Particles are driven by the action of the electric field on their electrophoretic mobilities [40]. Eqn. 4 shows the calculation of the electrophoretic mobility from the zeta potential of the colloid and the fluid viscosity. The zeta potential, or the electrokinetic potential, is determined by properties of the particle and of the fluid as shown:

$$
\zeta=\frac{q}{\varepsilon *\left(\frac{1}{r}+\frac{1}{\lambda_{D}}\right)}
$$


This calculation utilizes the surface charge of the particle, q, the electric permittivity constant, $\varepsilon$, the particle radius, $r$, and the Debye screening length, $\lambda_{\mathrm{D}}$. The Debye length is a value associated with the electric double layer of the solvent. It is a calculated value relating the properties of an electrolyte solution to the size of the double layer of a charged particle. This screening length is given by:

$$
\lambda_{D}=\left(\frac{\varepsilon * R T}{2 F^{2} z^{2} c}\right)^{\cdot 5}
$$

where $\varepsilon$ is the electric permittivity, $\mathrm{z}$ is the valence number of the electrolyte in solution and $\mathrm{c}$ is the concentration of the electrolyte. The values R, F, and $\mathrm{T}$ in the equation are the gas constant, $8.314\left(\mathrm{~J} / \mathrm{mol}^{*} \mathrm{~K}\right)$, Faraday's constant, $9.6487 \mathrm{e}^{4}(\mathrm{C} / \mathrm{mol})$, and the solution temperature in Kelvins, respectively.

Having determined the electrophoretic mobility of charged species the influence of the external electric field can be approached. This field is applied in the form of a voltage drop across the channel; the field strength is calculated by

$$
E=\frac{V}{h}
$$

where $\mathrm{V}$ is the voltage applied to the channel, and $\mathrm{h}$ is the dimension of the channel in the direction of the applied field. By combining the electrophoretic mobility and electric field strength, the movement as a result of the applied field is calculated

$$
Y_{e}=E * \mu * d t
$$

where $E$ is the magnitude of the applied electric field, $\mu$ is the electrophoretic mobility of the particle and dt is the time step during which motion occurs. 


\subsubsection{Velocity}

The component of the simulation responsible for driving the particles down the length of the channel is the fluid velocity. As previously stated, this model utilizes a laminar, parabolic flow profile. There are multiple potential approaches to determine the fluid influence on the particle; a common technique is utilizing the fluid pressure and the drag on the particle to calculate the velocity [41]. In this model it is assumed that particles follow the fluid streamlines on which their centroids reside. This assumption simplifies the solution of particle and fluid velocities, as the fluid velocity equals the particle velocity at that point. With this method it is only necessary to calculate the velocities of the particles. This technique was chosen in response to the complex geometries of channels in order to mitigate the computationally expensive velocity calculations. Unlike macro-scale fluidic system where round conduits and tubing are commonly utilized, microfluidic devices often have rectangular geometries (Figure 6). Largely this is a result of micro-manufacturing processes where etching and lithography are the preferred techniques. This rectangular geometry confounds the calculation of the velocity profile; unlike round channels where the velocity of any given point is a function of the radial distance from the center, this requires a complex summation to account for the $\mathrm{y}$ and $\mathrm{z}$ coordinates of the flow, assuming that flow is in the $\mathrm{x}$-direction [42]. Solutions to this velocity profile have been developed by two independent researchers: Pozrikidis and Bruus [43, 44]. 


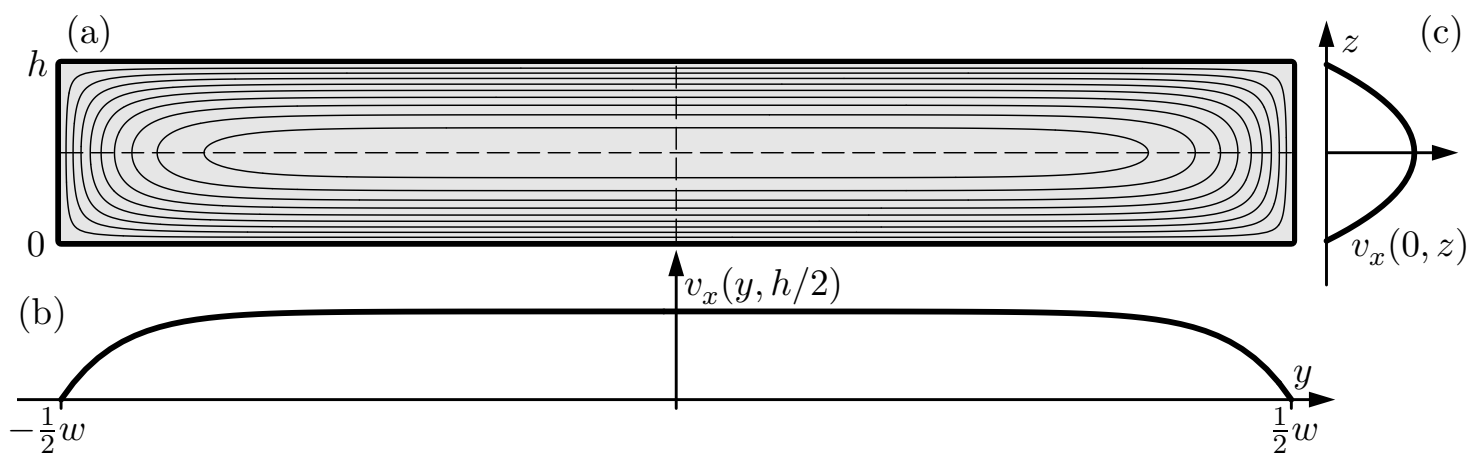

Figure 6 - Sketch of the velocity profile for laminar flow through a rectangular channel. a) Cross sectional view of the velocity distribution looking in the direction of flow; velocity is constant around the topographic tracings with higher velocities in the center and low velocity near the channel walls. b) Cross section looking across the flow in the $\mathrm{z}$-direction. c) Cross section of the flow looking from the y-direction [44].

The solutions from both researchers were included in the simulation; however, only one can be utilized at a time. The reason for the inclusion of both was to validate the accuracy of the system. Both remain included purely to demonstrate that two different approaches to the problem yield the same result. The Bruus solution is given:

$$
u_{x}(y, z)=\frac{48 Q}{\pi^{3} h w} * \frac{\sum_{n, o d d}^{\infty} \frac{1}{n^{3}}\left[1-\frac{\cosh \left(n \pi \frac{y}{h}\right)}{\cosh \left(n \pi \frac{w}{2 h}\right)}\right] \sin \left(n \pi \frac{Z}{h}\right)}{1-\sum_{n, o d d}^{\infty} \frac{192 h}{n^{5} \pi^{5} w} \tanh \left(n \pi \frac{w}{2 h}\right)}
$$

where $u_{x}$ is the fluid velocity at the location given by the coordinates $y, z$; the values $h$ and $w$ are the height and width of the rectangular channel, respectively. Q is the volumetric flow rate through the channel and $\mathrm{n}$ is the summation step. For the sake of computational brevity the summations were conducted to a finite value. Having determined the particle velocities they are converted to displacement by multiplying them by the time step and added particle motion phase. In this simulation the particle motion phase is limited to diffusive motion, electric field mediated motion and velocity. Having discussed the calculation of these components, the travel associated with the particle motion phase can be calculated and the particle locations updated in the model. 


\subsection{Particle Interactions}

The particle interaction phase of the model is comprised of the interplay between particles.

These reactions may result from direct contact between particles (collisions) or from proximity, which manifests itself as an interaction potential. One of the goals of this thesis was to implement a zero-overlap condition for particles in the model. The next sections relating to the particle interaction phase serve to accomplish this goal by modeling the particles as physical objects with a fixed volume. In conjunction with repulsion potentials and kinetic collision simulations these particle models will interact in a similar manner to that documented experimentally.

\subsubsection{Distance Calculations}

The first step in solving for inter-particle interactions is determining the pairwise distance between particles in the system. Once the distance is calculated the interplay between particles can be calculated. The pairwise distance between all particles is determined using the Euclidean distance calculation. The point-to-point distance, $\mathrm{d}$, in a three-dimensional system is given by

$$
d=\sqrt{\left(x_{2}-x_{1}\right)^{2}+\left(y_{2}-y_{1}\right)^{2}+\left(z_{2}-z_{1}\right)^{2}}
$$

where $\mathrm{x}, \mathrm{y}$, and $\mathrm{z}$ are Cartesian coordinates of particles. The indices 1 and 2 denote the colliding particles. In order to account for the surface-to-surface distances the particle radii, $r$, are added to Eqn. 17, as shown.

$$
d=\sqrt{\left(x_{2}-x_{1}\right)^{2}+\left(y_{2}-y_{1}\right)^{2}+\left(z_{2}-z_{1}\right)^{2}}-\left(r_{1}+r_{2}\right)
$$

Having established the inter-particle distances the information can be utilized to calculate collision interactions and reaction potentials.

Another key component of the distance calculation portion of the simulation is the enforcement of channel boundaries. As particles are driven through the modeled channel their diffusion and 
interactions bring them close to the system walls. Just as particles cannot be allowed to overlap other particles they cannot cross system boundaries. In order to prevent this, the distance between particles and walls are monitored. If a colloid approaches a wall a reaction is triggered in the collision system to prevent the species from passing through a wall. This distance is determined by

$$
d_{w a l l}=\left\{\begin{array}{c}
h-(z+r) \\
z-r \\
w-(y+r) \\
y-r
\end{array}\right.
$$

where $y$ and $\mathrm{z}$ are particle coordinates within the channel, $\mathrm{h}$ and $\mathrm{w}$ are the height and width of the channel. The distances $\mathrm{d}$ and $\mathrm{d}_{\text {wall }}$ are used in the next steps of the collision simulation.

\subsubsection{Kinetic Collisions}

In the case that the distance $d$ provided by the distance calculations is less than zero a kinetic collision is calculated between the interacting species. The development of this interaction step is based on all particle motion leading to the collision, namely the motion in the particle motion phase. When overlaps occur between particles they are treated as particle-particle collisions. Although it is reasonable to assume that a particle could collide simultaneously with multiple particles the simulation does not observe this case and each interaction is treated as a pair-wise collision. In a three dimensional system the collision of particles results in new velocity vectors and can result in a particle rotation [45]. In this model the particle collisions are considered frictionless, thereby eliminating the need for rotational motion terms in the algorithm[46]. It is assumed that the impact of particle rotation has a minimal, if any, effect on the accuracy of this simulation. The potential implications of particle rotation could include hydrodynamic effects on the fluid or additional momentum transfer to other particles during inter-particle interplay; however, in this simulation the particles do not impact the fluid flow, thereby negating any 
impacts that particle rotation could impose. On the topic of particle-particle interactions, rotation could impact the dynamics of the system; however, due to the scale of the system, it was assumed that such forces would be small compared with other contributions to particle motion.

The calculation of the three-dimensional colloidal collisions was accomplished with kinetic equation. In order to solve this system of equations the initial particle velocities were necessary; these were calculated from their displacement during the particle motion phase of the simulation, leading to the collision. The first equation to determine the collision response was the conservation of momentum, given by

$$
m_{1} \vec{u}_{2}+m_{2} \vec{u}_{2}=m_{1} \vec{v}_{2}+m_{2} \vec{v}_{2}
$$

where $\mathrm{m}$ is the particle mass, $\mathrm{u}$ is the initial velocity vector and $\mathrm{v}$ is the final velocity vector of particles 1 and 2. Next the coefficient of restitution was used to relate the initial and final velocities:

$$
\varepsilon=\frac{\vec{v}_{2}-\vec{v}_{1}}{\vec{u}_{1}-\vec{u}_{2}}
$$

where $\varepsilon$ is the coefficient of restitution, $\mathrm{u}$ is the initial velocity vector, and $\mathrm{v}$ is the final velocity vector for colliding particles 1 and 2. Due to the three dimensional motion of the particles the unit vector connecting the particles is necessary for the solution, given by

$$
\vec{e}=\frac{\vec{r}_{1}-\vec{r}_{2}}{\left|\vec{r}_{1}-\vec{r}_{2}\right|}=\frac{\vec{r}_{12}}{\left|\vec{r}_{12}\right|}
$$

where $r_{1}$ and $r_{2}$ are the particle location vectors and $r_{12}$ is the vector connecting the particles. By combining Eqns. 20, 21, and 22, the final particle velocities, $\mathrm{v}_{1}$ and $\mathrm{v}_{2}$, are found using the expressions 


$$
\vec{v}_{1}=\vec{u}_{1}-(1+\varepsilon) \frac{m_{2}}{\left(m_{1}+m_{2}\right)}\left(\vec{r}_{12} \cdot \vec{e}\right) \vec{e}
$$

and

$$
\vec{v}_{2}=\vec{u}_{2}+(1+\varepsilon) \frac{m_{1}}{\left(m_{1}+m_{2}\right)}\left(\vec{r}_{12} \cdot \vec{e}\right) \vec{e}
$$

These post-collision velocities are applied to particles by multiplying them by the time step to find the resulting displacement. These values are applied to the particle positions in the particle interaction phase, resulting in an updated location.

\subsubsection{DLVO Interactions}

In addition to the direct collisions between particles, the species interact through indirect contact in the form of attractive and repulsive potentials. These forces described by Derjaguin, Landau, Verwey, and Overbeek result in attraction at close distances and repulsion at larger separations (Figure 7). The general DLVO interaction energies for flat plates are shown in Eqns. 6 and 7; in order to account for the spherical geometry of the particles these functions must be adapted.

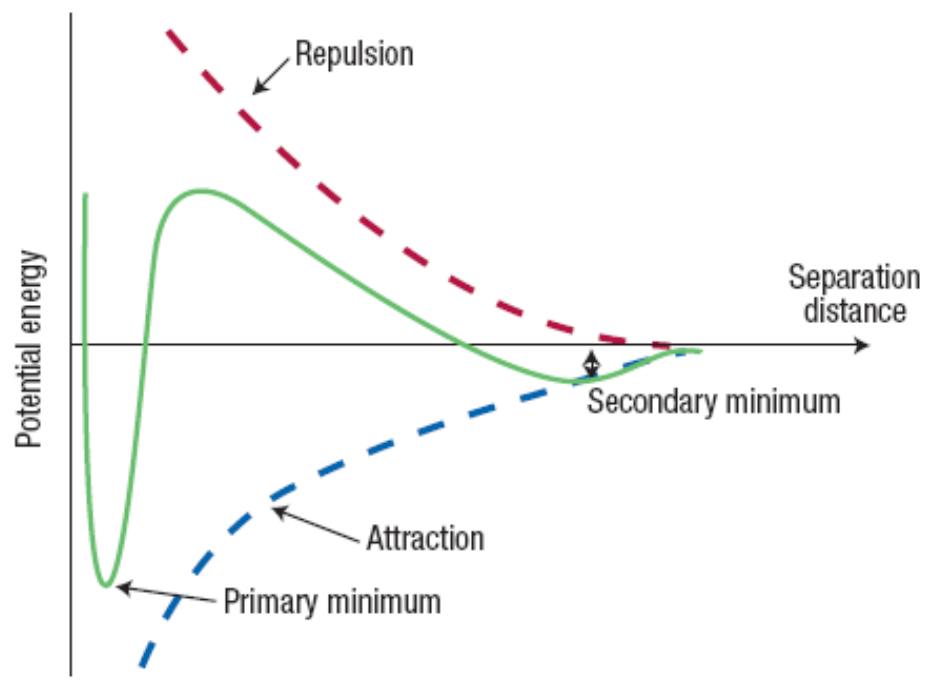

Figure 7 - Plot of DLVO Interaction Potential based on van der Waals attraction and electric double layer repulsion energies. At small separation distances interacting particles experience an attractive force; however at greater separations this becomes a repulsive potential. When the separation distance becomes sufficiently large the interaction potential approaches zero; this cutoff distance is typically 1 Debye length. 
Derjaguin researched and developed an approximation to account for different geometries in the calculation of interaction potentials; however his approximation has been modified to further account for the effects of particle curvature. By implementing this approximation into the Van der Waals and electrostatic double layer equations the interaction energies are predicted with higher accuracy than with the flat plate equations or Derjaguin's approximation (DA) alone [22]. The adaptation to the van der Waals potential in Eqn. 6 is given by

$$
U_{V D W}^{D A}=-\frac{A_{H}}{6 D} \frac{a_{1} a_{2}}{a_{1}+a_{2}}
$$

where $A_{H}$ is the Hamaker constant, $D$ is the surface-to-surface distance between spherical particles, and a is the radius of particles 1 and 2. The van Der Waals adaptation utilizes Derjaguin's approximation; however, the adapted calculation of the electric double layer potential utilizes Derjaguin's approximation with a Linear Superposition Approximation (LSA), given by modifying Eqn. 7 as shown:

$$
U_{E D L, L S A}^{D A}=64 \pi \varepsilon_{0} \varepsilon_{r} \gamma_{1} \gamma_{2} \frac{a_{1} a_{2}}{a_{1}+a_{2}}\left(\frac{k T}{z e}\right)^{2} \exp (-\kappa h)
$$

where $\mathrm{a}$ is the radius of particles 1 and 2. The gamma component of the double layer calculation relates the zeta potential of the particle to the valence charge of the electrolyte solution, given by

$$
\gamma=\tanh \left(\frac{z e \zeta}{4 k T}\right)
$$

where $\mathrm{z}$ is the electrolyte valence, e is the electron charge $1.602 \mathrm{e}^{-19} \mathrm{C}, \zeta$ is the zeta potential of the colloid, $\mathrm{k}$ and $\mathrm{T}$ are the Boltzmann constant and solution temperature, respectively. By combining the van der Walls and double layer forces the total interaction energy between the particles can be calculated as shown:

$$
U_{D L V O}=U_{V D W}+U_{E D L}
$$


where $\mathrm{U}_{\mathrm{VDW}}$ is the van der Waals potential calculated in Eqn. 26 and $\mathrm{U}_{\mathrm{EDL}}$ is the double layer potential calculated in Eqn. 27. This energy is converted to an electric field and, through the electrophoretic mobilities of the species the DLVO interaction manifests its influence. In order to determine which particles interact in this manner, a set of distances were selected for the Distance Calculations. The range of separation distances for participation in DLVO interactions is those greater than zero and less than the Debye length. Particles whose separation is greater than the Debye length will experience insignificant interaction potentials, suggesting that this is an acceptable cutoff distance.

Having established the criteria for inclusion in DLVO interactions and the calculation of the resulting potentials the values are used to update the particle locations.

\subsection{Parameters}

The previous sections discussed the design and physical calculations that compute the simulation; this section will focus on the user-defined parameters, specifically how they impact and contribute to the simulation. In any separation process the system is set up and calibrated for the procedure to ensure success. Similarly this model has user defined input variables that determine the outcome of the simulation. The first set of user inputs relate to the microfluidic device being simulated, specifically the geometry. This model assumes a rectangular geometry for the channel and allows specification of the dimensions. These dimensions play a key role in the physics of the separation as they impact the fluid velocity profile, hydrodynamic forces and length available for particle fractionation and diffusion.

The user also defines the fluid properties that directly impact the separation; these are the solvent viscosity, electrolyte valence, electrolyte concentration, temperature, and flow rate. By altering the fluid viscosity the diffusivity and zeta potential of particles are impacted. Temperature has a 
direct impact on diffusivity as well as on the surface potential used in DLVO calculations. The fluid properties relating to the electrolyte most directly impact the Debye length and, by association the inter-particle potentials influenced by electric double layer forces. Of the fluid properties selected by the user the flow rate has the greatest impact on simulation efficiency. High flow rates result in lower resolution and separation purity but must be sufficient to complete the simulation in a reasonable time frame.

The most influential parameter selected by the user is the voltage applied across the channel. This value determines, in conjunction with the channel height, the strength of the electric field. This field is largely responsible for the deflection and motion of particles resulting in species fractionation. The final system level input set by the user is the number of discrete time steps in the simulation. In order to ensure accuracy of the model it is necessary to select a sufficiently high number of time steps. Although a small number can be used for initial testing purposes a valid simulation requires a high number of time steps. By understanding the ramifications of electric field modification a separation can be tailored to produce a specific elution profile.

Having discussed the implications of the process-related parameters the properties of the particles will be examined. The user has the option to include one or two different particle species in the simulation, each with its own properties. These properties include: the number of particles simulated, particle radius, surface charge, electrophoretic mobility, particle density and particle mass. In the case of certain pairs of properties, mass-density and charge-mobility, only one of the values is needed as the other can be calculated based on other given information. The purpose of this redundancy is to allow for a wider range of potential particles to simulate, as not all properties may be readily available. By allowing the user to input the specific parameters of the sample particles, laboratory separations can be modeled. This allows validation of the 
simulation against experimental results and implicates the system for use in a design and optimization role for microfluidic separations.

\subsection{Simulation Configurations}

This model was developed with the functionality to model multiple separation configurations and fluid-particle interactions. This was accomplished by adjusting the initial placement of the particles. A simple fluid-particle system was developed by randomly seeding the particles throughout the channel. This allowed for validation of the velocity profile and ease of visualizing the particle-particle collisions and interactions. Another configuration simulates a Tfilter, where particles are injected into the upper half of the channel and, through diffusion and electric field mediated motion they disperse throughout the channel. T-filters are a wellcharacterized separation technique and this simulation allows comparison, again validating the systems efficacy. The most important simulation configuration represents free flow electrophoresis or electric field flow fractionation. This configuration initializes the sample at the top of the channel entrance.

Another simulation variation utilized in this model is continuous flow versus batch flow systems. Regardless of the separation type selected all parameters remain the same; the continuity is accomplished by re-initializing particles that have reached the end of the channel at the entrance. 


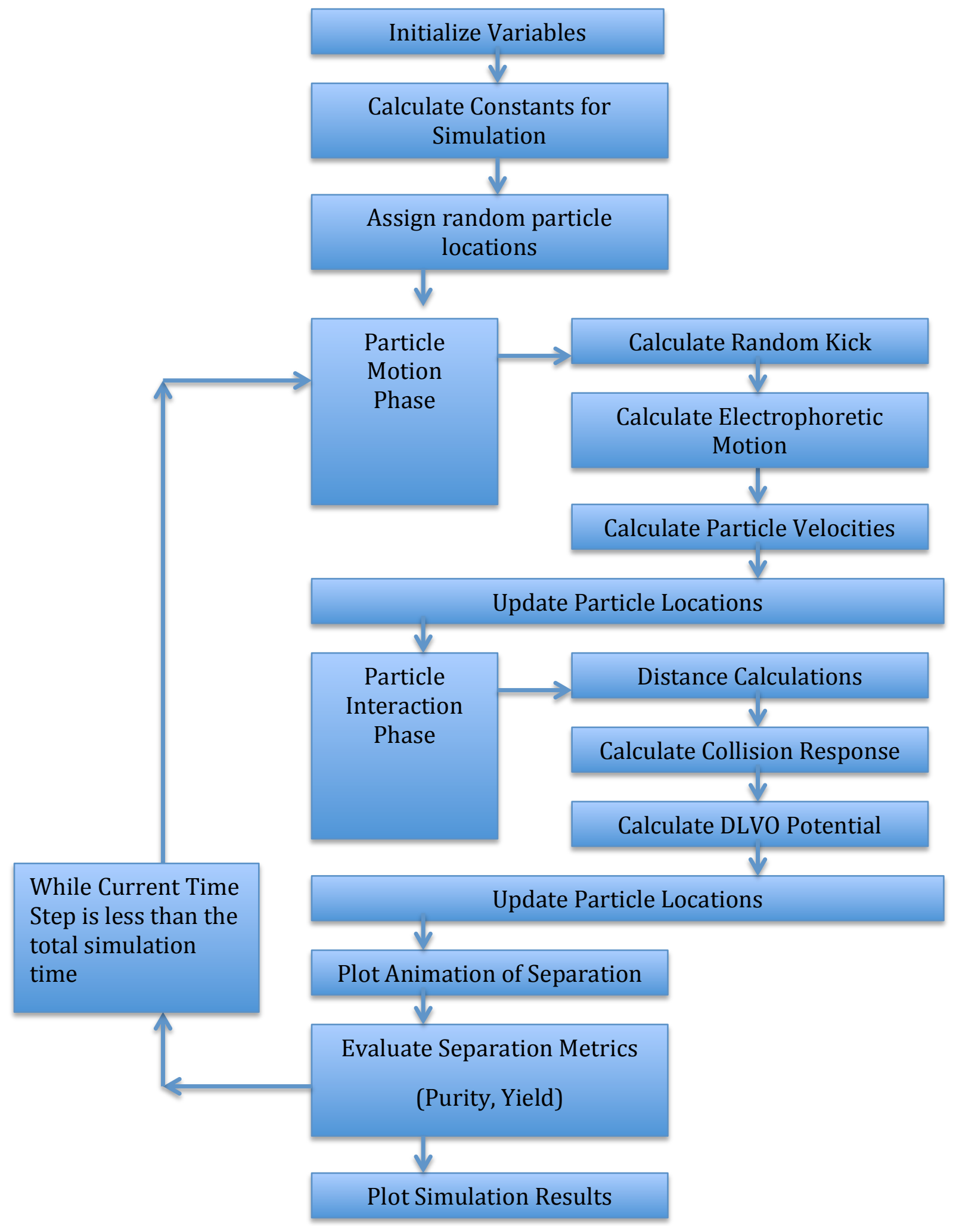

Figure 8 - Block diagram of the simulation algorithm. The model proceeds from top to bottom, looping from separation evaluation to the particle phase while the simulation is running 


\subsection{Limitations}

Although this model captures a majority of the physics to produce realistic results it has inherent limitations. Among the main drawbacks to this model is the nature of the fluid-particle coupling. The fluid dynamics utilize single direction coupling; the fluid acts on the particle but the particle does not exert and influence on the fluid. As a result some intricacies and phenomena noted in separation processes may be lost. The reason for the exemption of these hydrodynamic feedback mechanisms is the computational cost. In order to utilize two-way coupling between the particles and fluid the computation time would significantly increase and hardware limits would be approached if a sufficient number of particles were simulated.

Another limitation of this simulation lies with the use of finite time steps; it is possible that after collisions occur and particles are assigned new locations overlaps may exist. It is possible to resolve these overlaps with extended collision response algorithms however a rebounding situation can occur, resulting in a particle becoming trapped in a loop colliding between two nearby particles. Additionally the time required to resolve these secondary collisions would result in colliding particles to continue moving while other particles remain static, conflicting with the finite nature of each time step.

Lastly, typical fluid-particle simulations implement a sufficient number of particles to reach a volume fraction of 0.4 ; however, due to issues relating to computing capacity this is not feasible. Additionally, a volume fraction of that magnitude would have a significant influence on the hydrodynamics and without particle-fluid coupling such a particle concentration cannot be simulated accurately. 


\section{Testing}

After the design and development of the model, prior to a separation, the dynamic interactions within the computational simulation were tested for accuracy. This testing ensured that the physics utilized in the model was calculated properly and that the resulting fluid-particle and particle-particle interactions were correct. In order to evaluate the components of the model the components of the particle motion and interaction phases were tested individually. Following completion of the component testing the entire system can be evaluated in a separation simulation.

\subsection{System Validation}

The physics that this model was based on can be calculated theoretically using the equations shown in the previous chapter. In order to ensure that the physical functions produce correct values the computational outputs were compared to theoretical calculations or proven models, using the same inputs. The components tested in this manner were: diffusive motion, electric field mediated motion, fluid-particle velocity, collision detection, collision response, and DLVO interactions.

\subsubsection{Diffusive Motion}

The motion of particles from Brownian interactions was generated by random values in the three Cartesian directions, multiplied by the kick magnitude of the particle. To evaluate the validity of this random walk the root mean square value of a single particle's motion was calculated and plotted against simulation time. In order to obtain a high resolution the particle underwent $10,000,000$ time steps. The slope of the resulting plot should be six times the theoretical StokesEinstein diffusivity. For this test a particle radius of $27 \mathrm{~nm}$ was used in a solvent at $298 \mathrm{~K}$ with viscosity of 1e-3 Pa*s. 


\subsubsection{Electric Field Motion}

The particle motion resulting from electric field application is built into the simulation using the electrophoretic mobility in Eqn. 4. The electric-field mediated motion was taken from the simulation and compared to the theoretically calculated value. For this test a particle mobility of $1.2 \mathrm{e}-8 \mathrm{~m}^{\wedge} 2 / \mathrm{V}^{*} \mathrm{~s}$ was used with a voltage of 10 Volts across a $200 \mu \mathrm{m}$ channel.

\subsubsection{Velocity Profile}

As stated in the previous chapter two solutions of the flow profile were included in the simulation, primarily for test purposes. The Pozrikidis and Bruus (Eqn. 16) solutions were solved with the same input parameters to compare the results. Pozrikidis' solution to rectangular flow is given by

$$
u(y, z)=\frac{G}{2 \mu}\left[b^{2}-z^{2}+4 b^{2} \sum_{1}^{\infty} \frac{(-1)^{n}}{\alpha_{n}^{3}} \frac{\cosh \left(\alpha_{n} y / b\right)}{\cosh \left(\alpha_{n} a / b\right)} \cos \left(\alpha_{n} \frac{z}{b}\right)\right]
$$

where $u$ is the velocity at the channel locations given by coordinates $(y, z), G$ is the pressure drop across the length of the channel, and $\mu$ is the fluid viscosity [43]. The values a and $b$ correspond to one-half the channel width and height, respectively. The $\alpha_{n}$ component of this solution is related to the summation term, given by

$$
\alpha_{n}=(2 n-1) \pi / 2
$$

where $\mathrm{n}$ is the summation step in Eqn. 29. In order to find the calculate the pressure drop across the channel from the flow rate the following equation was used:

$$
Q=\frac{4 G a b^{3}}{3 \mu} F\left(\frac{a}{b}\right)
$$


where $Q$ is the volumetric flow rate, $\mathrm{G}$ is the pressure drop across the channel, $\mu$ is the fluid viscosity, and the values a and $\mathrm{b}$ are one-half the channel width and height. The function $\mathrm{F}($ ) is given

$$
F(x)=1-\frac{6}{x} \sum_{1}^{\infty} \frac{\tanh \left(\alpha_{n} x\right)}{\alpha_{n}^{5}}
$$

where $\alpha_{\mathrm{n}}$ is the term found in Eqn. 30, and $\mathrm{x}$ is the variable element of the equation; in the case of the use of this equation in Eqn. 31, the $x$ value equals $a / b$, the ratio of the channel width to height.

For this evaluation two separate experiments were conducted. First the two equations were solved with fixed parameters to directly compare the results. The fluid inputs for this solution were a flow rate of 10 Liters/minute and a viscosity of 1e-3 Pascal-seconds. The dimensions of this channel were $10 \mathrm{~mm}$ by $20 \mathrm{~mm}$, the particle locations are shown in the table below. The values were selected to give a representative sample of the velocities. Sets of particles were evaluated along the midline of the channel in the z-direction to represent the maximum velocity values. The other particle velocities were evaluated at points in the channel with sub-maximal values.

Table 1 - Coordinates of particles evaluated in the velocity profile testing. The channel these points reside in is $10 \mathrm{~mm}$ wide and a height of $20 \mathrm{~mm}$.

\begin{tabular}{|c|c|c|c|c|c|c|c|c|c|c|c|}
\hline \multicolumn{10}{|c|}{ Particle Coordinates and Velocity(m/s) in Flow Channel } \\
\hline $\mathrm{y}(\mathrm{mm})$ & 0 & 1 & 2 & 3 & 4 & 5 & 6 & 7 & 8 & 9 & 10 \\
\hline $\mathrm{z}(\mathrm{mm})$ & 10 & 10 & 10 & 10 & 10 & 10 & 12 & 14 & 16 & 18 & 20 \\
\hline
\end{tabular}

The second test conducted with the velocity was intended to generate representative flow profile of both solutions. 1000 random coordinate pairs were seeded into the equations; the resulting average velocities and plots should be similar. 


\subsubsection{Collision Detection}

The collision detection function feeds values to the collision response and DLVO interactions of the system. This is a crucial component and must function accurately. To test the accuracy of the collision detection a set of particles were initialized at known locations. Some of these particles will be in collisional contact while others will be within the interaction range for the DLVO potential. As the overall simulation is intended to model particles of varying size it is necessary to test the distance calculations between different sized species. This validation involved comparing the outputs of the collision detection function to the theoretically calculated Euclidean distances between pairs of particles. After the distances were calculated they were evaluated to determine if either a collision had occurred, the particles within one Debye length of each other, or of no interaction had happened. The input values fed into the system are shown in table 2, below.

Table 2 - Particle locations and radii necessary for distance calculations. The Debye length is used to determine whether particles are within the DLVO interaction range.

\begin{tabular}{|l|r|r|r|r|r|r|r|r|}
\hline \multicolumn{7}{|c|}{ Particle Properties for Distance Calculation Testing } \\
\hline Particle & 1 & 2 & 3 & 4 & 5 & 6 & 7 & 8 \\
\hline radius $(\mathrm{m})$ & $2.00 \mathrm{E}-06$ & $2.00 \mathrm{E}-06$ & $2.00 \mathrm{E}-06$ & $2.00 \mathrm{E}-06$ & $2.00 \mathrm{E}-06$ & $2.00 \mathrm{E}-06$ & $2.70 \mathrm{E}-08$ & $2.70 \mathrm{E}-08$ \\
\hline $\mathrm{x}(\mathrm{m})$ & 0.00051 & 0.00035 & 0.00099 & 0.00051 & 0.00025 & 0.00035 & 0.00099 & 0.00025 \\
\hline $\mathrm{y}(\mathrm{m})$ & $7.99 \mathrm{E}-06$ & $6.71 \mathrm{E}-05$ & $1.33 \mathrm{E}-05$ & $7.99 \mathrm{E}-06$ & $4.14 \mathrm{E}-05$ & $6.55 \mathrm{E}-05$ & $1.30 \mathrm{E}-05$ & $3.94 \mathrm{E}-05$ \\
\hline $\mathrm{z}(\mathrm{m})$ & $1.80 \mathrm{E}-05$ & 0.0002 & 0.00019 & $1.80 \mathrm{E}-05$ & $9.80 \mathrm{E}-05$ & 0.0002 & 0.00019 & $9.80 \mathrm{E}-05$ \\
\hline
\end{tabular}

\begin{tabular}{|l|l|}
\hline Debye Length $(\mathrm{m})$ & $3.04 \mathrm{E}-07$ \\
\hline
\end{tabular}

\subsubsection{Response}

Using the data generated from the collision detection test the collision response was tested to confirm that the model had correctly calculated the collision data. The initial motion leading to the collision of the colliding particles, shown in the table 3 , are random values in the same order of magnitude of the combined velocity, diffusion and electric field mediated motion components 
of the particles. The resulting outputs from the collision function were compared to the theoretical results, calculated using Eqns. 23 and 24 with a restitution coefficient of 1. Following the collision response the pairwise distances between particles was calculated to ensure that overlapping particles no longer existed.

Table 3 - Kinetic collision input data. The input velocity and mass of interacting particles are required in order to calculate the results from a collision.

\begin{tabular}{|l|r|r|r|r|r|r|r|r|}
\hline \multicolumn{7}{|c|}{ Motion and Mass of Particles Leading to Collision } \\
\hline Particle & 1 & 2 & 3 & 4 & 5 & 6 & 7 & 8 \\
\hline $\mathrm{dx}(\mathrm{m})$ & $1.00 \mathrm{E}-06$ & $2.00 \mathrm{E}-06$ & $3.00 \mathrm{E}-06$ & $4.00 \mathrm{E}-06$ & $5.00 \mathrm{E}-06$ & $6.00 \mathrm{E}-06$ & $7.00 \mathrm{E}-06$ & $8.00 \mathrm{E}-06$ \\
\hline $\mathrm{dy}(\mathrm{m})$ & $-1.00 \mathrm{E}-07$ & $1.00 \mathrm{E}-07$ & $-2.00 \mathrm{E}-07$ & $2.00 \mathrm{E}-07$ & $-3.00 \mathrm{E}-07$ & $-3.00 \mathrm{E}-07$ & $4.00 \mathrm{E}-07$ & $-4.00 \mathrm{E}-07$ \\
\hline $\mathrm{dz}(\mathrm{m})$ & $-1.00 \mathrm{E}-06$ & $1.00 \mathrm{E}-06$ & $-1.00 \mathrm{E}-06$ & $1.00 \mathrm{E}-06$ & $-2.00 \mathrm{E}-06$ & $2.00 \mathrm{E}-06$ & $-2.00 \mathrm{E}-06$ & $2.00 \mathrm{E}-06$ \\
\hline $\mathrm{m}(\mathrm{kg})$ & $3.35 \mathrm{E}-14$ & $3.35 \mathrm{E}-14$ & $3.35 \mathrm{E}-14$ & $3.35 \mathrm{E}-14$ & $3.35 \mathrm{E}-14$ & $3.35 \mathrm{E}-14$ & $8.24 \mathrm{E}-20$ & $8.24 \mathrm{E}-20$ \\
\hline
\end{tabular}

\subsubsection{DLVO Interactions}

Similar to the collision response testing, the evaluation of the DLVO function used the data from the collision detection test. In addition to the particle locations shown above, the data in the table below was used in the calculation of DLVO potentials. The DLVO function within the simulation was compared to the theoretically calculated particle interactions. 
Table 4 - Input data for calculating DLVO potentials. The gamma values are specific to the interacting particles; the remaining values relate to fluid properties or are universal constants.

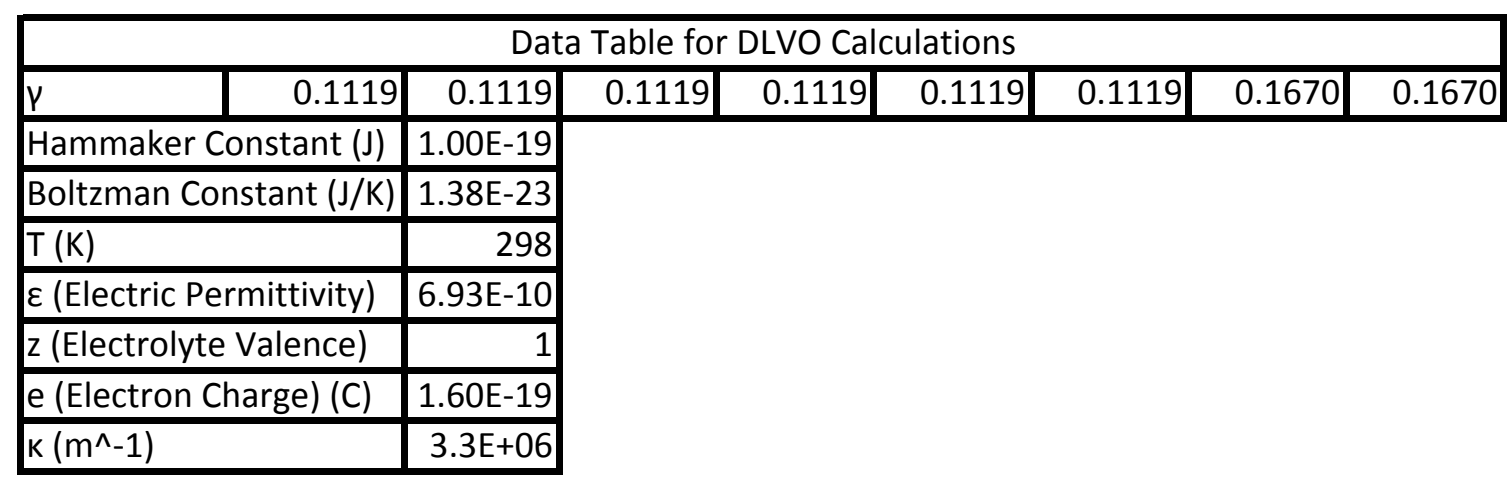

\subsection{Simulation of Microfluidic Separation}

Having tested the physics modules of the computational model it can be used to simulate a separation process. The specific separation that was modeled utilized a channel $500 \mu \mathrm{m}$ in length, $200 \mu \mathrm{m}$ in height and a width of $100 \mu \mathrm{m}$. The fluid viscosity was $1 \mathrm{e}-3 \mathrm{~Pa}^{*} \mathrm{~s}$ with an electrolyte concentration of $0.001 \mathrm{~mole} / \mathrm{m}^{\wedge} 3$ and a valence number of 1 . The temperature was $298 \mathrm{~K}$ and the particle properties are shown in the table below.

Table 5 - Particle properties input for this simulation

\begin{tabular}{|l|r|r|}
\hline \multicolumn{3}{|c|}{ Particle Properties } \\
\hline & Particle 1 & Particle 2 \\
\hline Radius $(\mathrm{m})$ & $2.00 \mathrm{E}-06$ & $2.70 \mathrm{E}-08$ \\
\hline Mobility $\left(\mathrm{m}^{\wedge} 2 / \mathrm{V}^{*} \mathrm{~s}\right.$ & $8.00 \mathrm{E}-09$ & $1.20 \mathrm{E}-08$ \\
\hline Density $\left(\mathrm{Kg} / \mathrm{m}^{\wedge} 3\right)$ & 1000 & 1000 \\
\hline
\end{tabular}

The applied voltage and flow rate were the variable elements in this experiment. Three different flow rates were tested, each with ten voltages. The purpose of this experiment is to generate a plot of separation efficiency as a function of flow rate and voltage. The flow rates and voltages used in the simulation are shown in the table below. 
Table 6 - Variable elements in this model; 3 flow rates were tested with 11 applied voltages. All other input values remained static during the simulation.

\begin{tabular}{|c|c|c|}
\hline \multicolumn{3}{|c|}{ Simulation Variables } \\
\hline Voltage (V) & \begin{tabular}{l|l|l|}
0 & 5 & 10
\end{tabular} & \begin{tabular}{l|l|l|l|l|l|l|l|}
15 & 20 & 25 & 30 & 35 & 40 & 45 & 50
\end{tabular} \\
\hline Flow Rate $(\mu \mathrm{L} / \mathrm{min})$ & 10 & \\
\hline
\end{tabular}

The separation was run for 10000 time steps with 1000 of each species. Particles were collected at different heights as they were eluted. Particle 1 was collected in the upper half while Particle 2 was collected in the lower half of the channel. The resulting fractionation was evaluated for purity and yield.

\section{Results}

\subsection{System Validation}

In order to evaluate the accuracy and validity of the separation model a series of tests were conducted. These tests first confirmed the calculations of the physics employed by the system to produce the simulation.

\subsubsection{Diffusive Motion Results}

Particles in solution diffuse freely by Brownian motion; this motion was characterized by the Stokes-Einstein diffusivity. The diffusive motion in the test revealed the particle motion shown in Figure 9. This plot does not quantitatively validate the diffusive properties of the particle but it does present an image of the randomness of particle motion. 


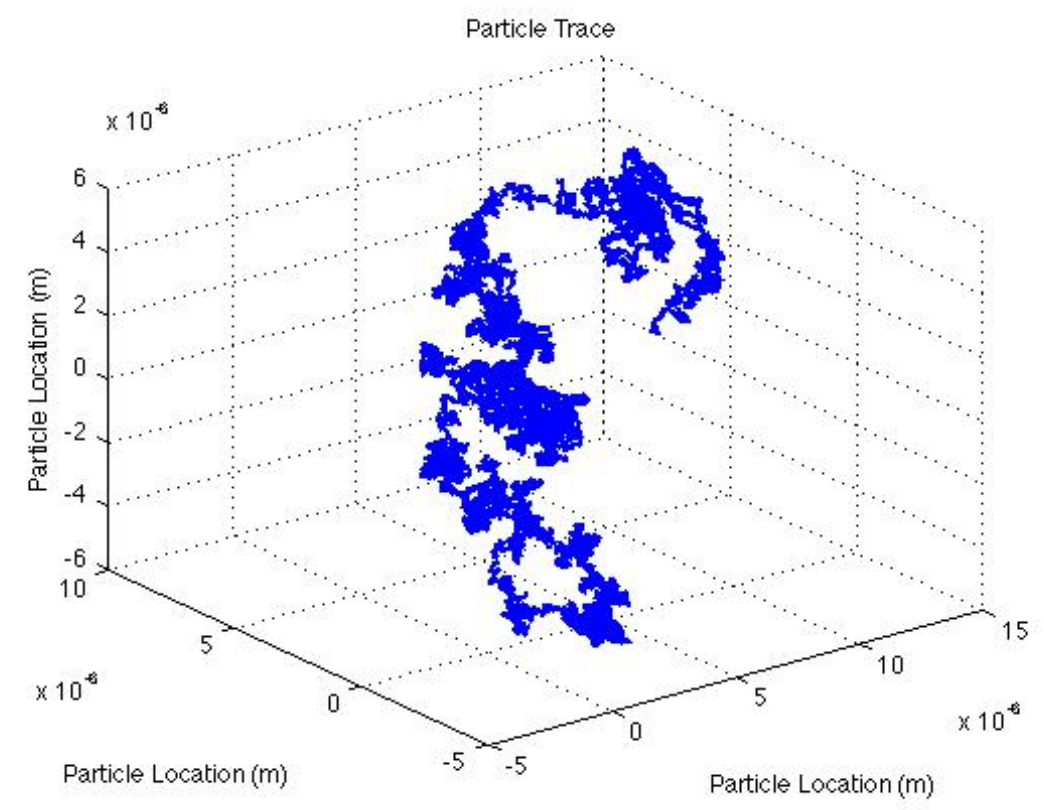

Figure 9 - Plot of particle motion due to Brownian motion over $10,000,000$ time steps.

The root-mean square plot of this motion is shown in Figure 10. The slope of this plot should be close to the theoretically calculated Stokes-Einstein value of $8.0815 \mathrm{e}-12 \mathrm{~m}^{2} / \mathrm{s}$. As shown on the plot the slope is $2.346 \mathrm{e}-11 \mathrm{~m}^{2} / \mathrm{s}$; by dividing this value by six a diffusivity of $7.66 \mathrm{e}-12 \mathrm{~m}^{2} / \mathrm{s}$ is

found. Although this value is not equal to the theoretical value the randomly generated diffusivity is sufficiently similar to confer the simulated diffusive motion of the particle as valid. 


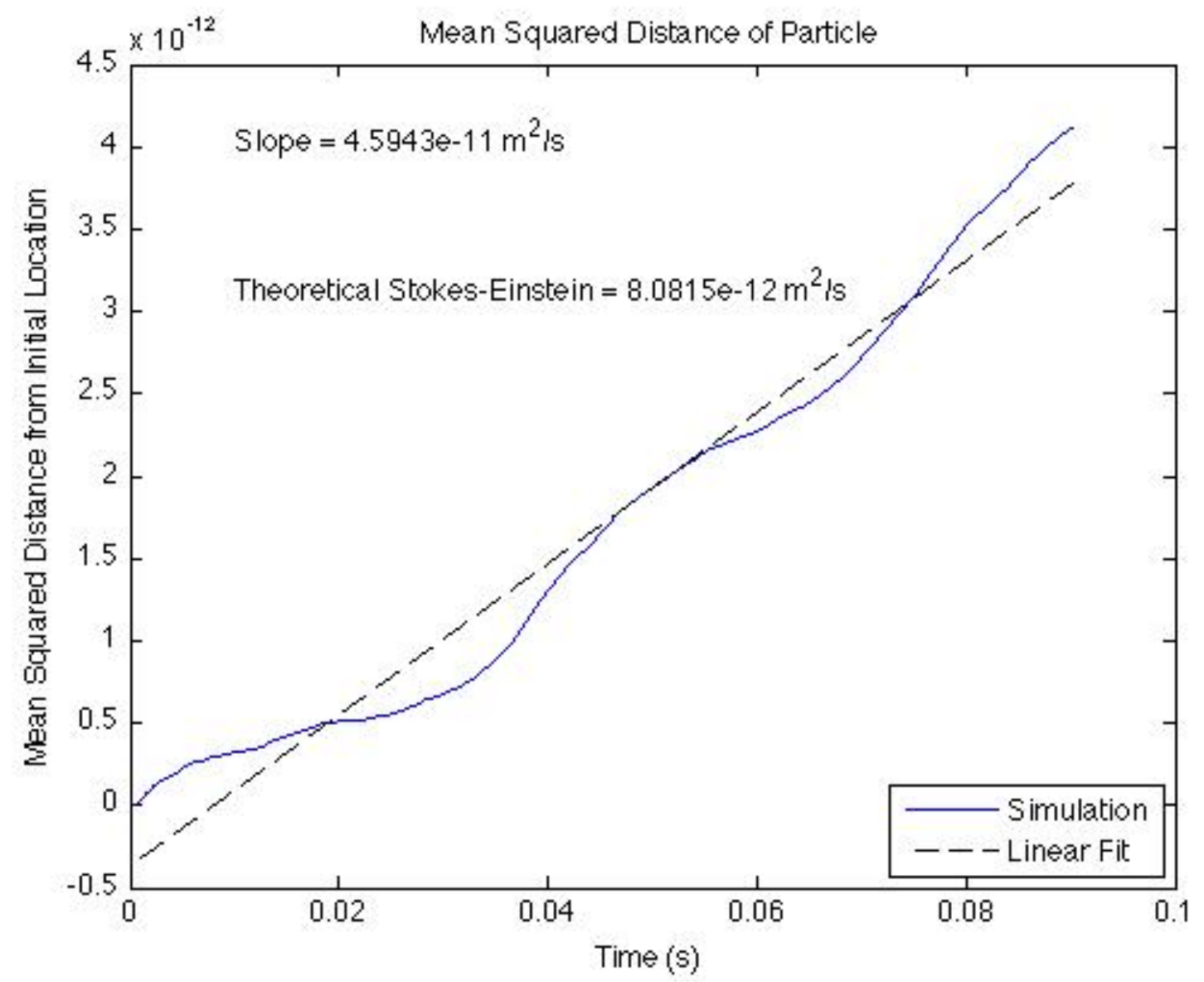

Figure 10 - Plot of the root-mean square values of the particle distance travelled by diffusive motion.

\subsubsection{Electric Field Motion Results}

The particle motion resulting from the application of an electric field is a relatively simple calculation; however for thoroughness the value computed in the model was compared to the theoretically calculated value, based on Eqn. 4. The shown in table 7 below confirm that the electric-field mediated particle motion is calculated correctly in the simulation. 
Table 7 - Comparison of the distances traveled as a function of the applied electric field for the simulated and theoretical calculations

\begin{tabular}{|l|r|}
\hline \multicolumn{2}{|l|}{ Electric Field Mediated Motion } \\
\hline Simulation $(\mathrm{m})$ & $-3.60 \mathrm{E}-05$ \\
\hline Theoretical $(\mathrm{m})$ & $-3.60 \mathrm{E}-05$ \\
\hline
\end{tabular}

\subsubsection{Particle and Fluid Velocity Results}

The testing of the fluid and particle velocity calculations consisted of the comparison of two separate derivations of the rectangular flow velocity profile. These solutions, by Pozrikidis and Bruus, were fed the same input values and the results shown in Table 8 were compared.

Table 8 - Particle coordinates and resulting velocities in the $x$-direction after calculation with the solutions by Bruus and Pozrikidis. The flow channel was $10 \mathrm{~mm}$ in width and $20 \mathrm{~mm}$ in height.

\begin{tabular}{|l|c|c|c|c|c|c|c|c|c|c|c|}
\hline \multicolumn{10}{|c|}{ Particle Coordinates and Velocity(m/s) in Flow Channel } \\
\hline $\mathrm{y}(\mathrm{mm})$ & 0 & 1 & 2 & 3 & 4 & 5 & 6 & 7 & 8 & 9 & 10 \\
\hline $\mathrm{z}(\mathrm{mm})$ & 10 & 10 & 10 & 10 & 10 & 10 & 12 & 14 & 16 & 18 & 20 \\
\hline Bruus & 0.000 & 0.030 & 0.054 & 0.070 & 0.080 & 0.083 & 0.078 & 0.064 & 0.042 & 0.017 & 0.000 \\
\hline Pozrikidis & 0.000 & 0.012 & 0.042 & 0.062 & 0.074 & 0.078 & 0.075 & 0.066 & 0.053 & 0.032 & 0.000 \\
\hline
\end{tabular}

As shown in the table the results from Bruus and Pozrikidis are not identical and, in some cases differ significantly. These discrepancies or errors can be may be accounted for in the nature of the sine and cosine summations in the solutions. As the solutions to the rectangular flow profile are not the same it is reasonable to assume that the resulting velocities may differ. Figure $11 \mathrm{a} \& \mathrm{~b}$, below, displays plots of the data in table 8 , showing similar flow profiles between the two solutions. Figure 11c\&d show the three-dimensional plots and average velocities of 1000 particles seeded into the equations. The average velocities shown in these plots, 0.0429 for Pozrikidis and 0.0456 for Bruus, are similar however, like the data in Table 8, show differences. Although the results are not identical they are sufficiently close to use these functions in the simulation. 

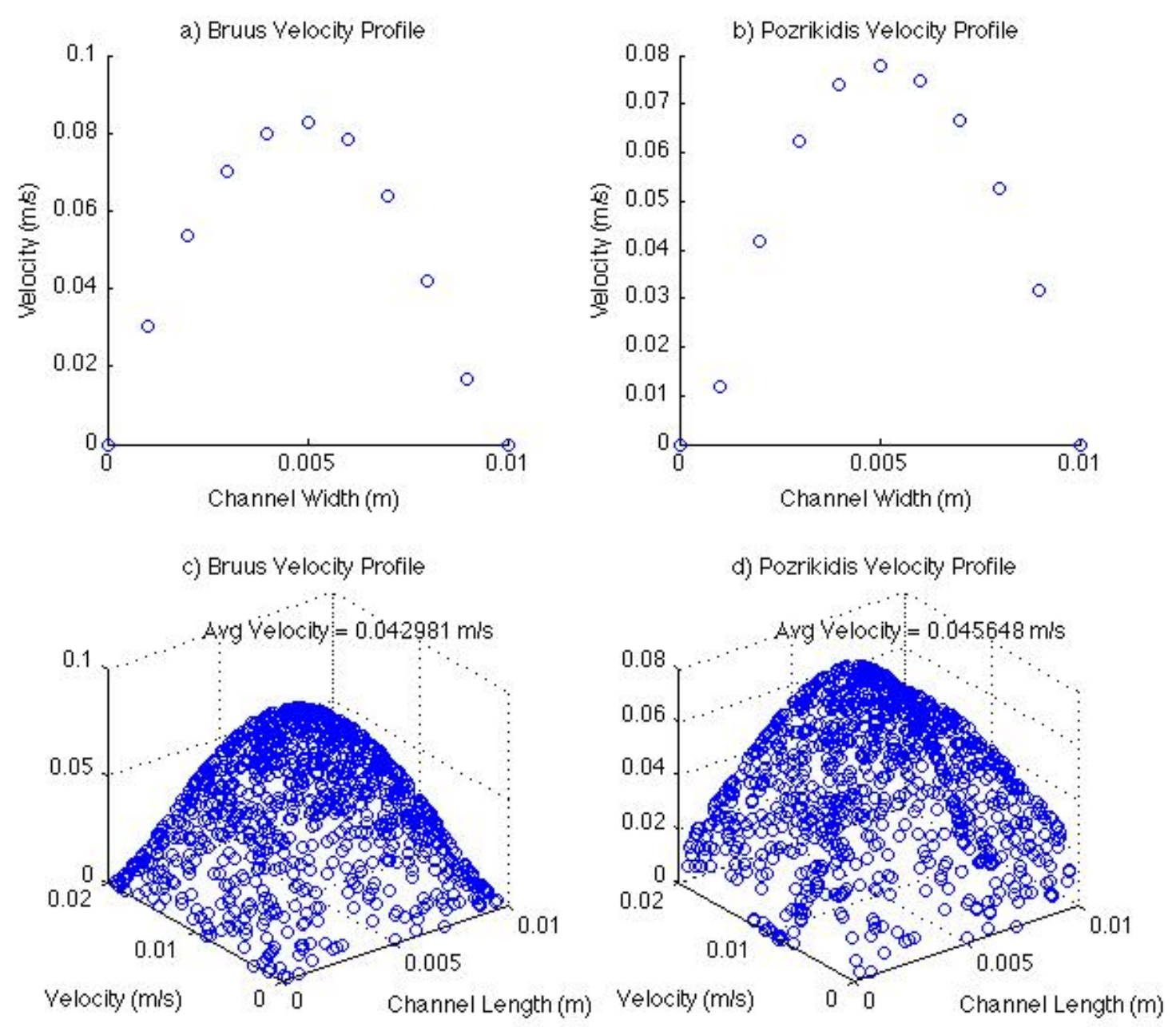

Figure 11 - Particle plots of the velocity profile for the Bruus and Pozrikidis solutions. a,b) The respective Bruus and Pozrikidis velocity results for the data in Table 8. c,d) Three dimensional velocity profile of 1000 random points in the respective Bruus and Pozrikidis equations, average particle velocities are shown above.

\subsubsection{Collision Detection Results}

The collision detection function takes the particle locations as inputs and determines the interaction type by calculating the Euclidean distance between the surfaces of particles. If collisions occur, this distance is less than zero and the function outputs the indices of the colliding pair of particles. If the pairwise interaction is not a collision but the separation distance 
is less than the Debye length the function outputs the indices of the particles interacting through DLVO potentials. The simulation determined that the pairs of particles shown in Table 9 have interactions and that all other combinations of particles do not interplay.

Table 9 - Indices of particles interacting through collisions or DLVO potentials, based on the particle locations and radii in Table 2.

\begin{tabular}{|c|c|c|}
\hline \multicolumn{3}{|c|}{ Indices of Colliding Particles } \\
\hline Collision Pair 1 & 2 & 6 \\
\hline Collision Pair 2 & 3 & 7 \\
\hline \multicolumn{3}{|c|}{$\begin{array}{c}\text { Indices of Particles Interacting through } \\
\text { DLVO Potentials }\end{array}$} \\
\hline DLVO Pair 1 & 1 & 4 \\
\hline DLVO Pair 2 & 5 & 8 \\
\hline
\end{tabular}

These pairs of points were compared to the theoretically calculated Euclidean distance between particles, shown below in Table 10. This table shows the distance between all pairs of particles. Only the upper triangle is displayed to because of matrix symmetry. By taking the indices of collisions from the distance function results and finding the corresponding distance on Table 10, both pairs of colliding particles have a distance of less than 0 . Similarly this applies to the DLVO pairs; the distances corresponding to the interacting particles are less than the Debye length of $3.04 \mathrm{e}-7 \mathrm{~m}$ but greater than 0 . This validates the distance calculation function. 
Table 10 - Pairwise distance between all particles in Table 2. The rows and columns refer to the particle indices.

\begin{tabular}{|r|r|r|r|r|r|r|r|r|}
\hline \multicolumn{8}{|c|}{ Theoretical Pairwise Distance Between Pairs of Particles } \\
\hline & 1 & 2 & 3 & 4 & 5 & 6 & 7 & 8 \\
\hline 1 & 0 & 0.00024 & 0.00050 & 0.00000 & 0.00027 & 0.00025 & 0.00050 & 0.00027 \\
\hline 2 & 0 & 0 & 0.00063 & 0.00024 & 0.00014 & $-1.4 \mathrm{E}-07$ & 0.00064 & 0.00014 \\
\hline 3 & 0 & 0 & 0 & 0.00050 & 0.00073 & 0.00064 & $-1.4 \mathrm{E}-07$ & 0.00073 \\
\hline 4 & 0 & 0 & 0 & 0 & 0.00026 & 0.00024 & 0.00051 & 0.00026 \\
\hline 5 & 0 & 0 & 0 & 0 & 0 & 0.00013 & 0.00074 & 0.00000 \\
\hline 6 & 0 & 0 & 0 & 0 & 0 & 0 & 0.00064 & 0.00014 \\
\hline 7 & 0 & 0 & 0 & 0 & 0 & 0 & 0 & 0.00074 \\
\hline 8 & 0 & 0 & 0 & 0 & 0 & 0 & 0 & 0 \\
\hline
\end{tabular}

\subsubsection{Collision Results}

Having verified the distance calculation function the collision response was tested using the

collisions predicted within the function.

Table 11 - Particle motion resulting from simulated collision interactions. The colliding pairs of particles leading to these results were 2-6, and 3-7.

\begin{tabular}{|l|r|r|r|r|r|r|r|r|}
\hline \multicolumn{7}{|c|}{ Motion of Particles Following Collision - Simulation } \\
\hline Particle & 1 & 2 & 3 & 4 & 5 & 6 & 7 & 8 \\
\hline $\mathrm{d} x(\mathrm{~m})$ & 0 & $5.15 \mathrm{E}-06$ & $3.00 \mathrm{E}-06$ & 0 & 0 & $2.85 \mathrm{E}-06$ & $-5.51 \mathrm{E}-07$ & 0 \\
\hline $\mathrm{dy}(\mathrm{m})$ & 0 & $1.57 \mathrm{E}-06$ & $-2.00 \mathrm{E}-07$ & 0 & 0 & $-1.77 \mathrm{E}-06$ & $-1.15 \mathrm{E}-06$ & 0 \\
\hline $\mathrm{dz}(\mathrm{m})$ & 0 & $1.07 \mathrm{E}-06$ & $-1.00 \mathrm{E}-06$ & 0 & 0 & $1.93 \mathrm{E}-06$ & $-2.96 \mathrm{E}-06$ & 0 \\
\hline
\end{tabular}

Table 12 - Calculated particle motion resulting from collision interactions of particles 2-6, and 3-7.

\begin{tabular}{|l|r|r|r|r|r|r|r|r|}
\hline \multicolumn{7}{|c|}{ Motion of Particles Following Collision - Theoretical } \\
\hline Particle & 1 & 2 & 3 & 4 & 5 & 6 & 7 & 8 \\
\hline $\mathrm{dx}(\mathrm{m})$ & 0 & $5.15 \mathrm{E}-06$ & $3.00 \mathrm{E}-06$ & 0 & 0 & $2.85 \mathrm{E}-06$ & $-5.51 \mathrm{E}-07$ & 0 \\
\hline $\mathrm{dy}(\mathrm{m})$ & 0 & $1.57 \mathrm{E}-06$ & $-2.00 \mathrm{E}-07$ & 0 & 0 & $-1.77 \mathrm{E}-06$ & $-1.15 \mathrm{E}-06$ & 0 \\
\hline $\mathrm{dz}(\mathrm{m})$ & 0 & $1.07 \mathrm{E}-06$ & $-1.00 \mathrm{E}-06$ & 0 & 0 & $1.93 \mathrm{E}-06$ & $-2.96 \mathrm{E}-06$ & 0 \\
\hline
\end{tabular}

Following the applied particle motion from the collision, the Collision Detection function was run again, determining that no particles had a negative separation distance. 


\subsubsection{DLVO Interaction Results}

Similar to the collision reactions the DLVO test used the results of the collision detection as inputs, as well as the data in Table 4. The DLVO function was run for these interacting pairs and the potential was computed, the results shown in Table 13 below. The DLVO potential for the interacting pairs of particles was computed theoretically as well, with the results shown on the right of the table. The computed potentials match the theoretical potentials, indicating that the DLVO computation within the simulation is correct.

Table 13 - The calculated and simulated DLVO potential between interacting particles is shown in the table above. The interaction is attractive if the energy is negative and repulsive if the energy is positive.

\begin{tabular}{|l|r|r|r|r|}
\hline \multicolumn{4}{|c|}{ DLVO Potential in Pairs of Interacting Particles } \\
\cline { 2 - 5 } & \multicolumn{2}{|c|}{ Simulation } & \multicolumn{2}{c|}{ Theoretical } \\
\cline { 2 - 5 } & Pair 1 & Pair2 & Pair1 & Pair2 \\
\hline van der Waals Potential (J) & $-2.51 \mathrm{E}-18$ & $-1.53 \mathrm{E}-21$ & $-2.51 \mathrm{E}-18$ & $-1.53 \mathrm{E}-21$ \\
\hline EDL Repulsion $(\mathrm{J})$ & $1.13 \mathrm{E}-18$ & $1.76 \mathrm{E}-20$ & $1.13 \mathrm{E}-18$ & $1.76 \mathrm{E}-20$ \\
\hline DLVO Potential $(\mathrm{J})$ & $-1.39 \mathrm{E}-18$ & $1.61 \mathrm{E}-20$ & $-1.39 \mathrm{E}-18$ & $1.61 \mathrm{E}-20$ \\
\hline
\end{tabular}

\subsection{Separation Results}

Having established that the simulated physics of the model function the system as a whole was evaluated in a series of separation experiments. The results of this evaluation are shown in Figures 12, 13, and 14. Figure 12 displays the yield and purity of small and large particles as a function of voltage. The flow rate for this separation was $5 \mu \mathrm{L} / \mathrm{min}$. Yield and purity were determined for the particles collected as they eluted from the channel. Particles were collected based on the height of their elution; large particles were collected in the upper half and small particles in the lower half. 


\section{Results at Flow Rate $\mathrm{Q}=5 \mathrm{e}-6 \mathrm{~L} / \mathrm{mir}$}
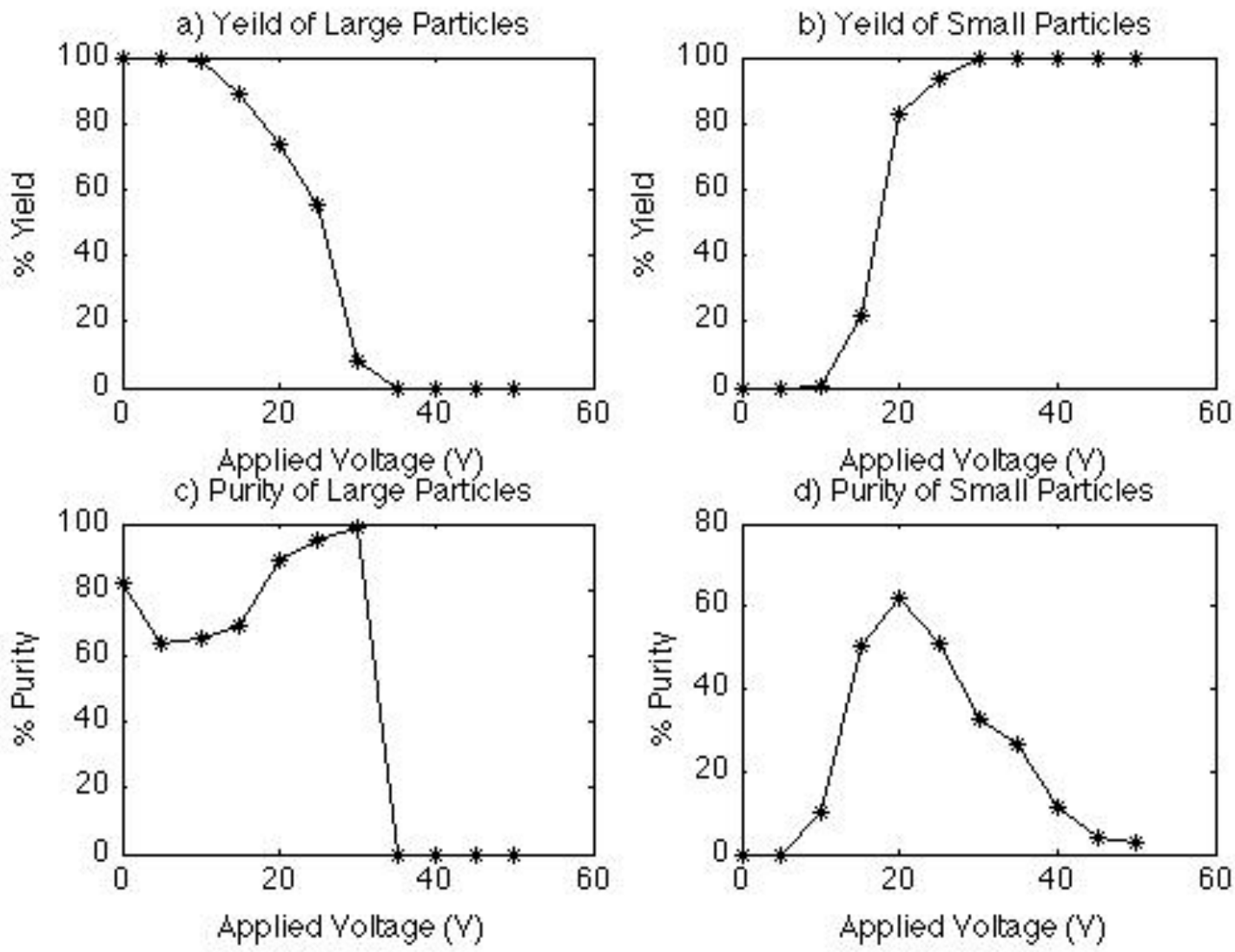

Figure 12 - Separation results from flow rate of $5 \mathrm{e}-6 \mathrm{~L} / \mathrm{min}$ at variable voltages ranging from 0-50 volts. The voltage was applied over a 200-micron channel to generate the electric field. a) Percent yield of large particles (radius 2.6 microns) b) Percent yield of small particles (27nanometers) c) Purity of large particles d) Purity of small particles

Figure 13 shows the simulation results when the flow rate is increased to $10 \mu \mathrm{L} / \mathrm{min}$. For this dataset the same voltages were applied so a relationship can be drawn between the flow rates and separation efficiencies. 


\section{Results at Flow Rate $\mathrm{Q}=10 \mathrm{e}-6 \mathrm{~L} / \mathrm{mir}$}
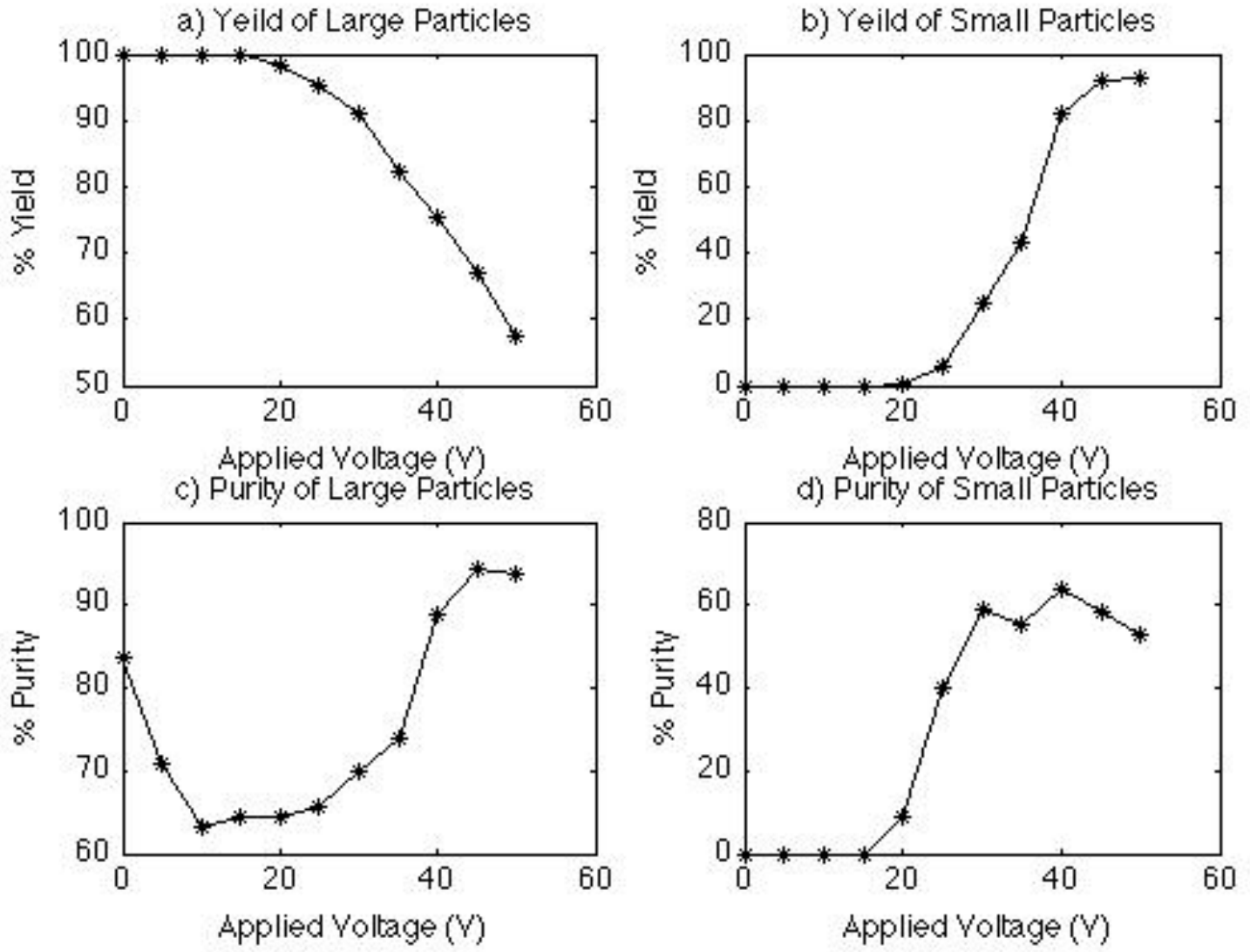

Figure 13 - Separation results from flow rate of $10 \mathrm{e}-6 \mathrm{~L} / \mathrm{min}$ at variable voltages ranging from $0-50$ volts. The voltage was applied over a 200-micron channel to generate the electric field. a) Percent yield of large particles (radius 2.6 microns) b) Percent yield of small particles (27nanometers) c) Purity of large particles d) Purity of small particles

Figure 14 displays the results from the separation with the same parameters as the previous simulations, but with a flow rate of $15 \mu \mathrm{L} / \mathrm{min}$. 

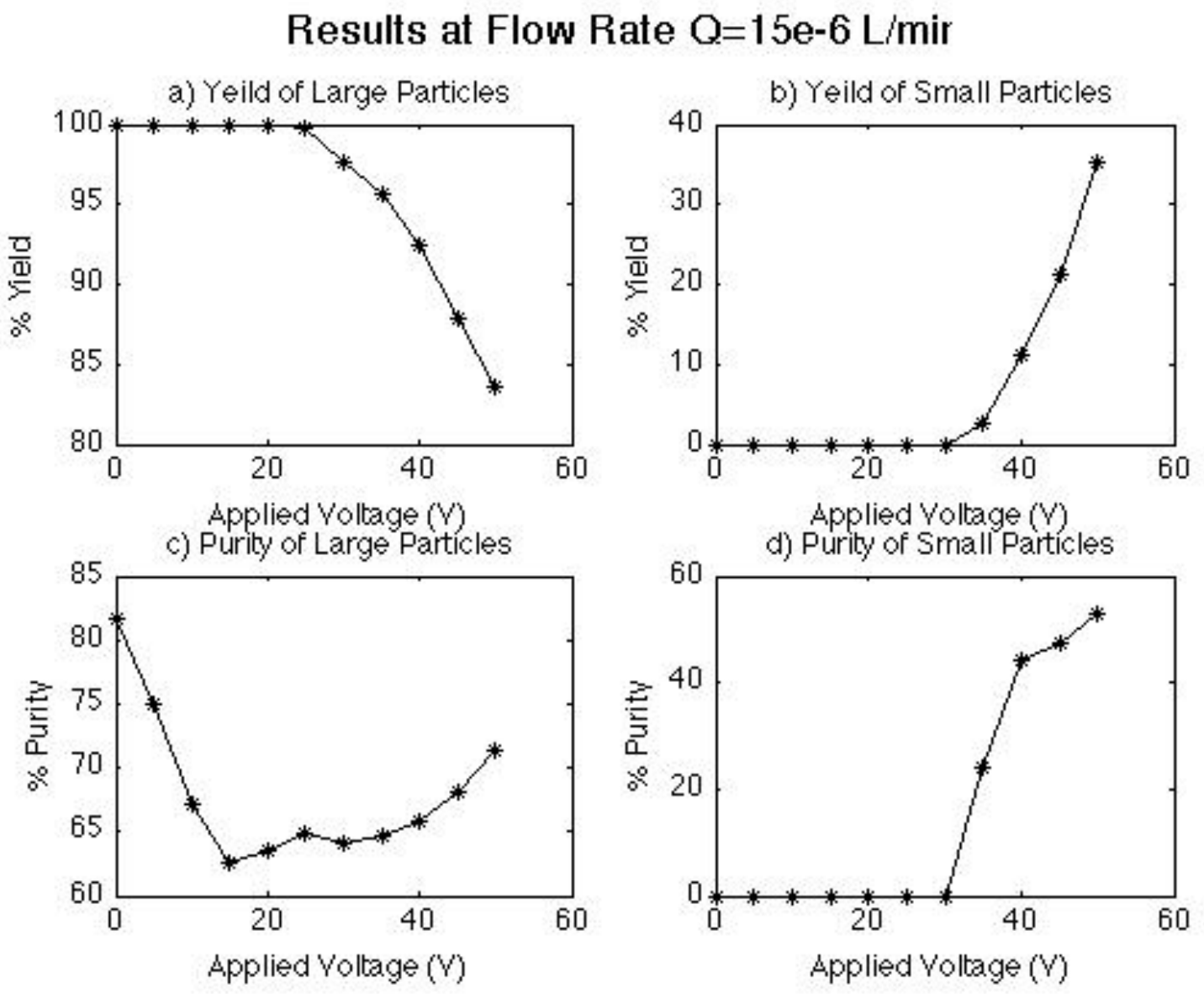

Figure 14 - Separation results from flow rate of $15 \mathrm{e}-6 \mathrm{~L} / \mathrm{min}$ at variable voltages ranging from $0-50$ volts. The voltage was applied over a 200-micron channel to generate the electric field. a) Percent yield of large particles (radius 2.6 microns) b) Percent yield of small particles (27nanometers) c) Purity of large particles d) Purity of small particles

\section{Discussion}

The objective of this thesis was to develop a computationally modeled separation process that captures sufficient physics in the fractionation of particles to evaluate the separation of likecharged particles in a continuous flow environment. By accomplishing these goals the model could be used to optimize separations and design improved systems. The simulation was evaluated through a series of experiments to confirm its validity. The first set of tests confirmed its ability to capture the physics of the separation process through diffusion, electric-field driven particle motion, fluid velocity, particle collisions and DLVO interactions. The tests for these 
physical aspects of the system demonstrated that the model accurately computes the dynamic components of particle motion. The data illustrating this conclusion is found in the results section of this thesis.

Having established that the simulation models the individual components of a separation process the system as a whole was tested. This experiment demonstrated the impacts of fluid flow rate through the channel and the voltage applied to produce the electric field. By tailoring these values to the separation the yield and purity can be optimized. As shown in figure 12, the large particles have the highest yield and purity when no voltage is applied and at a flow rate of $5 \mu \mathrm{L} / \mathrm{min}$, whereas the small particles have no yield. This separation profile is related to hydrodynamic chromatography, where the larger particles sample higher fluid velocity streams due to their size and elute sooner. As this simulation was run for a finite time only the particles that eluted were counted; if the simulation time were extended the purity of large particles would decrease, as a higher number of small particles would eventually elute. By examining a higher voltage, 20 volts for example, the yield of large particles has dropped while the yield and purity of small particles has significantly increased. This is due to the electric field mediated motion driving the particles toward the bottom of the channel. The small particles have a higher electrophoretic mobility than the large particles so they elute at a lower average height than the large particles. It is important to note that as the yield of large particles decrease the purity approaches $100 \%$. This phenomenon occurs when the electric field is sufficient to propel the majority of the small particles below the collection height for the large particles. As the voltage is further increased the number of large particles in the lower half of the channel increase, decreasing the purity of the small sample collection. 
The results shown in figure 13 follow the same trend however, due to the elevated flow rate of $10 \mu \mathrm{L} / \mathrm{min}$, the peaks are shifted toward the higher end of the applied voltages. If the experiment were continued for higher voltages the plots would follow the same trend. This same principle applies for the results shown in Figure 14 where the experiment was conducted at a flow rate of $15 \mu \mathrm{L} / \mathrm{min}$. It should be noted that in Figures 12 and 13, the peaks occur at 20volts and 40 volts, respectively. This seems to have a direct correlation to the flow rate of the fluid and particles, $5 \mu \mathrm{L} / \mathrm{min}$ in Figure 12 and $10 \mu \mathrm{L} / \mathrm{min}$ in Figure 13. These plots illustrate the relationship between flow rate and electric field magnitude on the separation of particles, however if higher purity and yield are necessary than those available other properties of the system must be adapted.

Other key parameters of the system that could be changed to alter the simulation results include the fluid, the channel dimension, and the height cutoff that divides large particle collection from small. By adapting this collection cutoff height between the particles the separation results can be shifted to better select for a specific species. This optimal separation height can be determined by monitoring the average elution heights of the particle species and, by examining the standard deviation of heights within the channel a collection cutoff can be placed to optimize the collection of one or both species. A limitation of tuning the simulation in this manner is the impact on the yield of small particles. If the collection height is lowered and the electric field increased to shift the average particle height toward the bottom of the channel, the purity of small particles can be increased in addition to both the purity and yield of large particles. A drawback to this method is the significantly decreased yield of small particles; similar to hydrodynamic chromatography they are delayed in the low flow region near the channel wall [47]. Although the particles may be in the correct height range to be collected, they fail to elute from the channel during the simulated timeframe. If the simulation were to be continued for a 
sufficient time following injection of the sample the small species would eventually be collected and increase the yield.

Due to the dimensions of the system it may not be feasible to manufacture the divider between the eluting species if the cutoff height is too close to the bottom or top of the channel. An alternative to this is changing the channel dimensions. By increasing the height of the channel the distance across which to fractionate the particles is heightened. Based on the electric field and flow magnitudes this could benefit the yield and purity. Another alternative is changing the channel length, increasing the exposure to the electric field. Dimensional changes to the system can improve separations of specific particles; however, these are design changes that require new microfluidic devices to be built.

To demonstrate the impact of dimensional changes another separation experiment was conducted. This test utilized a channel with twice the length and a flow rate of $10 \mu \mathrm{L} / \mathrm{min}$; due to the extended length and exposure of particles to the electric field a lower range of field magnitudes was used. In Figure 13 the maximum yields occurred at 40 volts; for this experiment it was assumed that the channel length has a similar relationship to separation results as flow rate so the electric field was produced with 20 volts across the channel height. The results from this experiment are shown in Figure 15 below.

This data demonstrates that by changing channel dimensions in addition to tuning voltage and flow rate inreased yield and purity are possible. Compared to Figures 12 and 13 where high purity came at the cost of low yields this experiment suggests that both can be obained. Looking at Figure $15 \mathrm{a}, \mathrm{c}$ the large particles reach a maximum purity around 20 volts and the yield at that point is $79 \%$. Compared to Figure 13 where the maximum combined purity and yield was $90 \%$ 
and $66 \%$ respectively, this is a significant increase. Shifting focus to the small particles the maximum purity obtained in this configuration was $66 \%$ with a yield of $97 \%$. This purity is not high however the yield is sufficient that further processing could be pursued to increase the purity.

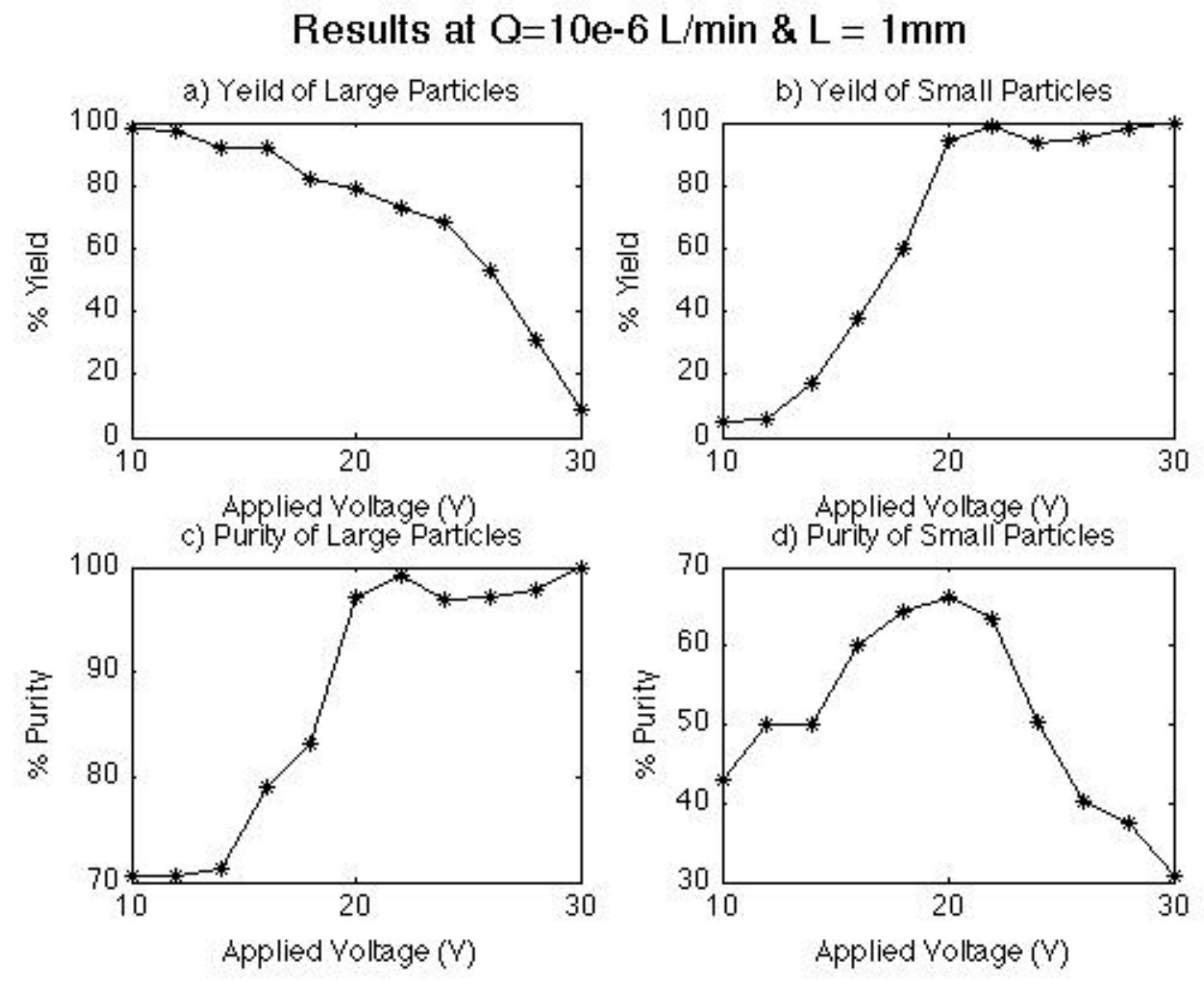

Figure 15 - The separation results from a simulation with extended channel length, L. The flow rate for this fractionation was 10 microliters/minute at a variable voltage ranging from 10-30. a) Percent yield of large particles (radius 2.6 microns) b) Percent yield of small particles (27nanometers) c) Purity of large particles d) Purity of small particles

During the testing of the physical components of the system it was found that the result of collision interactions may place particles within one Debye length of others; the current state of the system accounts for this resulting interaction by computing the distance between particles after collisions and feeding the new interactions to the DLVO function. A limitation of this 
method lies with the potential for DLVO interactions to result in collisions. This possibility is not accounted for in the simulation; due to the finite duration of each time step it is not realistic to resolve these rebounding reactions between particles. A potential solution to this would be to model the collisions and interactions on a system scale rather than as pairwise interplay, however this would require higher processing capabilities.

By utilizing computational models such as these separation processes can be optimized to match the target species in a biological sample. These optimizations can improve the resulting yield and purity from the fractionation process, reducing the need for additional sample processing. This type of simulation is also useful in the design and testing of separation systems prior to manufacture. By customizing and validating the microfluidic device for specific samples prior to building the system the resulting design will be better suited for its task.

\section{Conclusion}

Improved computational models of microfluidic separations would help drive the field of clinical diagnostics and aid in increasing the utility of existing devices. In this thesis a computational simulation of a fractionation process was developed to examine the separation of like charged particles in a continuous flow environment was feasible, while maintaining high yield and purity. To determine that the model would accurately represent the physics of separation processes, computational experiments were conducted to ensure validity. These experiments are described and the results shown in the testing and results portions of this report. To optimize the simulation in Matlab ${ }^{\circledR}$ the simulation code was developed in a vectorized form to minimize looping structures. While this improves computing efficiency for small numbers of particles $(<5000)$, the system is unable to process higher numbers in a timely manner. Another goal of 
this thesis was to eliminate particle overlap in the simulation. This was accomplished by implementing a collision response and interaction potential between particles either in contact or in close proximity. In the data produced and discussed in the results and conclusion it was shown that both high yield and purity can be achieved by optimizing dimensions, flow rated, and electric field magnitude. The experiments conducted demonstrated that while high separation efficiency can be obtained for one species the yield or purity of the other might be sacrificed. By customizing the channel dimensions to maximize the difference in average heights of the particles, high metrics can be achieved for both species in a separation.

Future directions for this research include adapting the model to a parallel computing platform. With increased processing capacity, higher particle numbers could be simulated. This would increase the validity of the simulation by more closely modeling physiological concentrations. With the increased computing capacity, Stokesian dynamics could be implemented, providing two-way coupling between the particle and fluid. This addition to the simulation would improve the simulated hydrodynamics. If higher particle concentrations are used then the Stokesian dynamics are more important as the elevated total volume of particles will have and increased impact of the fluid flow. This model should, in the future, be used in parallel with microfluidic separation experiments to verify that the data generated does in fact simulate experimental data and to use the model to optimize separation processes. 


\section{References:}

1. Thomas H. Schulte, R.L.B., Bernhard H. Weigl, Microfluidic technologies in clinical diagnostics. Clinica Chimica Acta, 2002(321): p. 1-10.

2. Kurt E. Petersen, W.A.M., Gregory T. A. Kovacs, M. Allen Northrup, Lee A. Christel, Farzad Pourahmadi, Toward Next Generation Clinical Diagnostic Instruments: Scaling and New Processing Paradigms. Biomedical Microdevices, 1998. 1(1): p. 71-79.

3. Bernhard H. Weigl, R.L.B., Catherine R. Cabrera, Lab-on-a-chip for drug development. Advanced Drug Delivery Reviews, 2003. 55: p. 349-377.

4. Andrew J. de Mellow, N.B., Dealing with 'real' samples: sample pre-treatement in microfluidic systems. The Royal Society of Chemistry, 2003(3): p. 11-19.

5. Jan Lichtenberg, N.F.d.R., Elisabeth Verpoorte, Sample pretreatment on microfabricated devices. Talanta, 2002. 56: p. 233-266.

6. R.F. Probstein, Physicochemical Hydrodynamics: An Introduction. Second ed2003, Hoboken, New Jersey: John Wiley \& Sons, Inc.

7. Todd M. Squires, S.R.Q., Microfluidics: Fluid physics at the nanoliter scale. Reviews of Modern Physics, 2005. 77(3): p. 977-1026.

8. Rohit Karnik, S.H., Huanan Zhang, Ying Mei, Daniel . Anderson, Jeffrey M. Karp, and Robert Langer, Nanomechanical control of cell rolling in two dimensions through surface patterning of receptors. Nano Letters, 2008. 8(4): p. 1153-1158.

9. Wesley C. Chang, L.P.L., Dorian Liepmann, Biomemetic technique for adhesion-based collection and separation of cells in a microfluidic channel. The Royal Society of Chemistry, 2004(5): p. 64-73.

10. N. Pamme, Continuous flow separations in microfluidic devices. The Royal Society of Chemistry, 2007(7): p. 1644-1659.

11. Marko T. Blom, E.C., R. Edwin Oosterbroek, Rob Tijssen, Albert van den Berg, OnChip Hydrodynamic Chromatography Separation and Detection of Nanoparticles and Biomolecules. Analytic Chemistry, 2003. 75(24): p. 6761-6768.

12. Emil Chmela, R.T., Marko T. Blom, Han J. G. E. Gardeniers, Albert van den Berg, A Chip System for Size Separation of Macromolecules and Particles by Hydrodynamic Chromatography. Analytic Chemistry, 2002(74): p. 3470-3475.

13. K.D.Altria, Overview of capillary electrophoresis and capillary electrochromatography. Journal of Chromatography, 1999. A(856): p. 443-463.

14. Dirk Janasek, M.S., Andreas Manz, Joachin Franzke, Electrostatic induction of the electric field into free-flow electrophresis devices. The Royal Society of Chemistry, 2006. 6: p. 710-713.

15. Daniel E. Raymond, A.M., H. Michael Widmer, Continuous Sample Pretreatment Using a Free-Flow Electrophoresis Device Integrated onto a Silicon Chip. Analytic Chemistry, 1994. 66: p. 2858-2865.

16. Pierluigi Reschiglian, A.Z., Barbara Roda, Elisa Michelini, Aldo Roda, Field-flow fractionation and biotechnology. TRENDS in Biotehnology, 2005. 23(9): p. 475-483.

17. T.N. Shendruk, G.W.S., Operational-modes of field-flow fractionation in microfluidic channels. Journal of Chromatography, 2012(1233): p. 100-108.

18. Fathi A. Messaud, R.D.S., J. Ray Runyon, Tino Otte, Harald Pasch, S. Kim Ratanathanawongs Williams, An overview on field flow fractionation techniques and 
their applications in the separation and characterization of polymers. Progress in Polymer Science, 2009.

19. Bruce K. Gale, K.D.C., A. Bruno Frazier, A Micromachined Electrical Field-Flow Fractionation System. IEEE Transactions on Biomedical Engineering, 1998. 45(12): p. 1459-1469.

20. Niem Tri, K.C., Ronald Beckett, Development of Electrical Field-Flow Fractionation. Analytic Chemistry, 2000(72): p. 1823-1829.

21. V.L. Per Linse, Electrostatic Attraction and Phase Separation in Solutions of LikeCharged Colloidal Particles. Physical Review Letters, 1999. 83(20): p. 4208-4211.

22. Subir Bhattacharjee, M.E., Michal Borkec, DLVO Interaction between Colloidal Particles: Beyond Derjaguin's Approximation. Croatica Chemica Acta, 1998. 71(4): p. 883-903.

23. J. Regtmeier, R.E., T.T. Duong, P. Reimann, D. Anselmetti, A. Ros, Pulsed-field separation of particles in a microfluidic device. European Physics Journal, 2007. E(22): p. 335-340.

24. David R. Foss, J.F.B., Structure, diffusion, and rheology of Brownian suspensions by Stokesian Dynamics simulation. Journal of Fluid Mechanics, 2000. 407: p. 167-200.

25. Thomas Bugener, D.K., Hans J. Herrmann, Simulation of particle mixing in turbulent channel flow due to intrinsic fluid velocity fluctuation. Computational Physics, 2011: p. 1-9.

26. Al Diez, A.O., J Garcia de la Tore, Brownain dynamics simulation of analytical ultracentrifugation experiments. BMC Biophysics, 2011. 4(6): p. 1-7.

27. P. S. Grassia, E.J.H., L. C. Nitsche, Computer simulations of Brownian motion of complex systems. Journal of Fluid Mechanics, 1995. 282: p. 373-403.

28. Russel, W.B., Brownian Motion Of Small Particles Suspended In Liquids. Annual Review of Fluid Mechanics, 1981. 13: p. 425-455.

29. Scott H. Northrup, H.P.E., Kinetics of protein-protein association explained by Brownian dynamics computer simulation. Biochemistry, 1992. 89: p. 3338-3342.

30. Ermak, McCammon, Ermak-McCammon equation, 1978.

31. M. Ripoll, K.M., R. G. Winkler, G. Gompper, Dynamic regimes of fluids simulated by multiparticle-collision dynamics. Physical Review, 2005(72): p. 1-14.

32. Anatoly Malevanets, R.K., Mesoscopic model for solvent dynamics. Journal of Chemical Physics, 1999. 110(17): p. 8605-8613.

33. Anatoly Malevanets, R.K., Solute molecular dynamics in a mesoscale solvent. Journal of Chemical Physics, 2000. 112(16): p. 7620-7269.

34. T. Ihle, D.M.K., Stochastic rotation dynamics. I. Formalizm, Galilean invariance, and Green-Kubo relations. Physical Review, 2003. E(67): p. 1-11.

35. Nobuhiko Watari, M.M., Norio Kikuchi, Ronald G. Larson, Masao Doi, Simulation of DNA motion in a microchannel using stochastic rotation dynamcis. Journal of Chemical Physics, 2007. 126(094902): p. 1-7.

36. M.J. Clifton, Numerical simulation of protein separation by continuous-flow electrophoresis. Electrophoresis, 1993(14): p. 1284-1291.

37. S. Krishnamoorthy, Z.J.C., J.J. Feng, Simulation-Based Analysis of Dielectrophoretic Field Flow Fractionation Devices. IEEE Design \& Test of Computers - Biochips, 2007: p. 50-58. 
38. R. Kapral, Multiparticle Collision Dynamics: Simulation Of Complex Systems On Mesoscales. Advances in Chemical Physics, 2008. 140: p. 89-146.

39. Thanh N. Phung, J.F.B., Georges Bossis, Stokesian Dynamics simulation of Brownian suspensions. Journal of Fluid Mechanics, 1996. 313: p. 181-207.

40. N. G. Douglas, A.A.H., H. R. C. Pratt, G. W. Stevens, Electrophoretic Mobilities of Proteins and Protein Mixtures. Chemical Engineering Science, 1995. 50(5): p. 743754.

41. Martin Frank, D.A., Eric R. Weeks, Jeffrey F. Morris, Particle migration in pressuredriven flow of a Brownian suspension. Journal of Fluid Mechanics, 2003. 493: p. 363378.

42. Rui Lima, S.W., Shuji Tanaka, Motohiro Takea, Takuji Ishikawa, Ken-ichi Tsubota, Yohsuke Imai, Takami Yamaguchi, In vitro blood flow in a rectangular PDMS microchannel: experimental observations using a confocal micro-PIV system. Biomedical Microdevices, 2008. 10(2): p. 153-167.

43. C. Pozrikidis, Introduction to Theoretical and Computational Fluid Dynamics1997, Oxford, New York: Oxford University Press.

44. H. Bruus, Theoretical Microfluidics, in Department of Micro and Nanotechnology, T.U.o. Denmark, Editor 2005.

45. Olaf Herbst, M.H., Annette Zippelius, Dynamics of inelastically colliding spheres with Coulomb friction: Relaxation of translational and rotational energy. Granular Matter, 2000. 2: p. 211-219.

46. Patric Muller, T.P., Oblique impact of frictionless spheres: on the limitations of hard sphere models for granular dynamics. Granular Matter, 2012. 14: p. 115-120.

47. L. Pasol, M.M., M.L. Ekiel-Jezewska, E. Wajnryb, J. Blawzdziewicz, F. Feuillebois, Motion of a sphere parallel to plane walls in a Poiseuille flow. Application to field-flow fractionation and hydrodynamic chromatography. Chemical Engineering Science, 2011. 66: p. 4078-4089. 


\section{Appendix A: Computational Model}

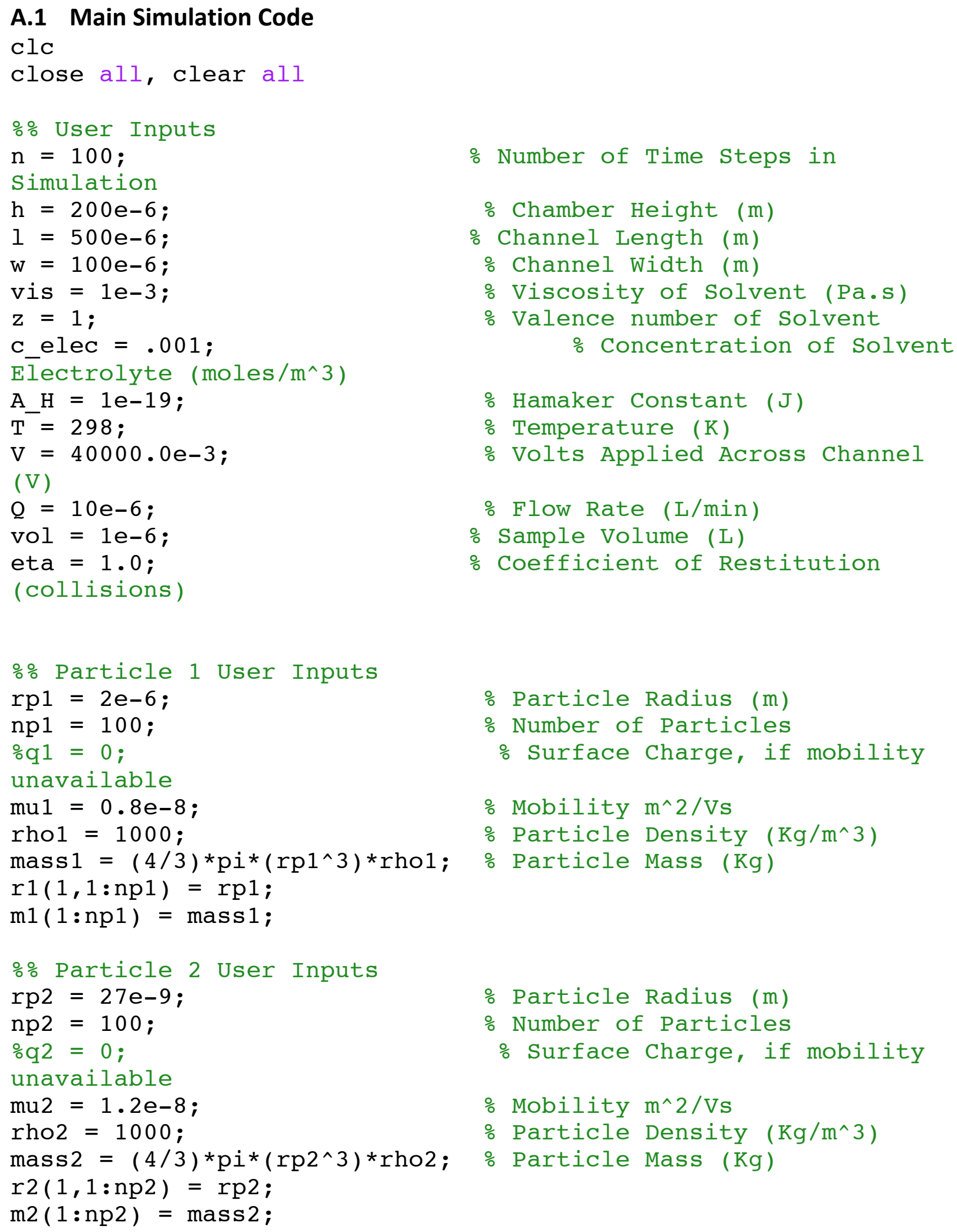


\% Constants

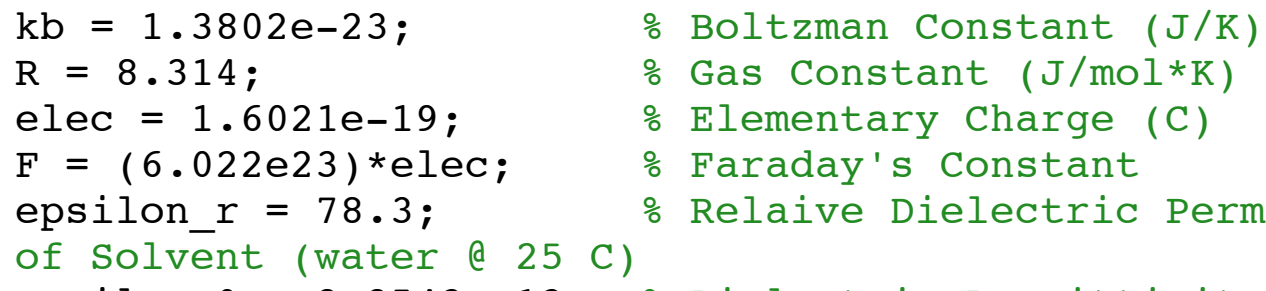




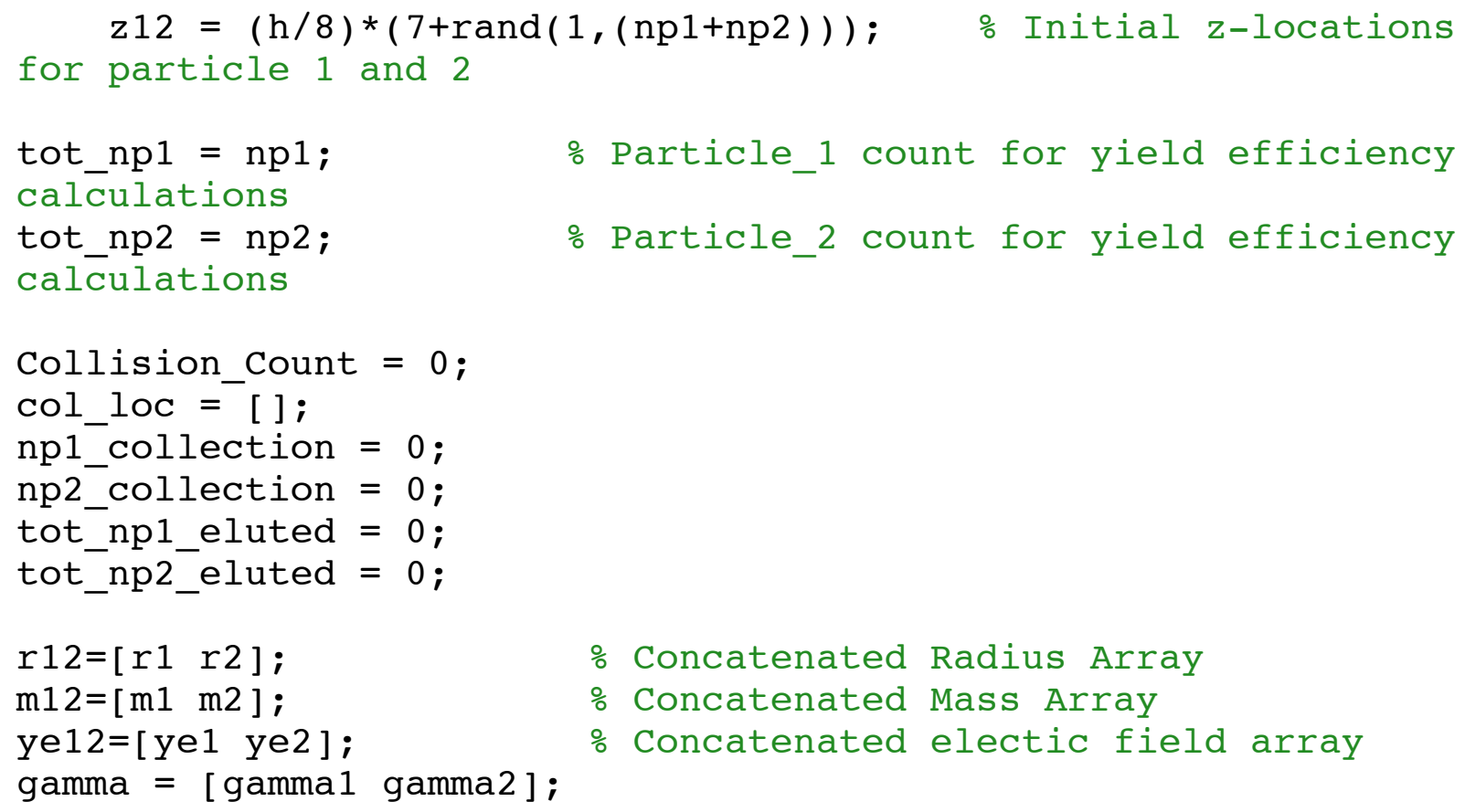




$$
\text { ye12=[ye1 ye2 }] \text {; }
$$

\% Concatenated E-Field component

\begin{abstract}
$\mathrm{x}_{-}$vel = velocity $(\mathrm{Q}, \mathrm{vis}, \mathrm{w}, \mathrm{h}, \mathrm{y} 12, \mathrm{z} 12)$;
。 $\mathrm{x}$ _vel = Vel_Function $(Q, \mathrm{w}, \mathrm{h}, \mathrm{y} 12, \mathrm{z} 12)$;

from Bruus, Mortensen, Lima

\% Pozrikidis

\% Velocity Function
\end{abstract}

$$
\begin{aligned}
& \mathrm{dx} 12=\mathrm{dx} 12+\mathrm{dt} * \mathrm{x} \text {-vel; } \% \text { Particle motion in } \mathrm{x} \text {-direction } \\
& \mathrm{dz} 12=\mathrm{dz} 12+\mathrm{ye} 12 ; \quad \% \text { Particle motion in y-direction } \\
& \mathrm{x} 12=\mathrm{x} 12+\mathrm{dx} 12 ; \quad \text { o Updated } \mathrm{x} \text {-locations for } \mathrm{x} \text {-direction } \\
& \text { y12 = y12 + dy12; } \quad \text { o Updated y-locations for } y \text {-direction } \\
& \mathrm{z} 12=\mathrm{z} 12+\mathrm{dz} 12 ; \quad \text { o Updated } \mathrm{y} \text {-locations for z-direction }
\end{aligned}
$$

을 Particle Interactions

[ r c ri ci D] = Collision_Detection $(\mathrm{x} 12, \mathrm{y} 12, \mathrm{z} 12, \mathrm{r} 12$, lambda_D $)$;

Collision_Count=Collision_Count+length $(r)$; $\quad$ Total number of Collisions

$[\mathrm{x} 12, \mathrm{y} 12, \mathrm{z} 12]=$ Collision $(\mathrm{x} 12, \mathrm{y} 12, \mathrm{z} 12, \mathrm{dx} 12, \mathrm{dy} 12, \mathrm{dz} 12, \mathrm{~m} 12$, $r, c, d t$, eta, r12);

o Calculates the collision interactions of particles

if isempty(ri)

$[\mathrm{x} 12, \mathrm{y} 12, \mathrm{z} 12]=\operatorname{DLVO}\left(\mathrm{x} 12, \mathrm{y} 12, \mathrm{z} 12, \mathrm{r} 12, \mathrm{~m} 12, \mathrm{ri}, \mathrm{Ci}, \mathrm{A} \_\mathrm{H}, \mathrm{D}\right.$, gamma, epsilon, kappa, kb, T, $z$, elec, dt);

end

$\begin{array}{ll}\mathrm{y} 12((\mathrm{y} 12+\mathrm{r} 12)>\mathrm{w})=\mathrm{w}-\mathrm{r} 12((\mathrm{y} 12+\mathrm{r} 12)>\mathrm{w}) ; & \text { \% Wall Boundaries } \\ \mathrm{y} 12((\mathrm{y} 12-\mathrm{r} 12)<0)=\mathrm{r} 12((\mathrm{y} 12-\mathrm{r} 12)<0) ; & \text { \% Wall Boundaries } \\ \mathrm{z} 12((\mathrm{z} 12+\mathrm{r} 12)>\mathrm{h})=\mathrm{h}-\mathrm{r} 12((\mathrm{z} 12+\mathrm{r} 12)>\mathrm{h}) ; & \text { \% Wall Boundaries } \\ \mathrm{z} 12((\mathrm{z} 12-\mathrm{r} 12)<0)=\mathrm{r} 12((\mathrm{z} 12-\mathrm{r} 12)<0) ; & \text { \% Wall Boundaries }\end{array}$

eluted $=$ find $(x 12>1) ; \quad \%$ Particles that elute from channel np1_eluted $=$ eluted $($ eluted $<=$ np1); $\%$ Indices of species 1 that eluted

np2_eluted $=$ eluted $($ eluted $>$ np1); $\%$ Indices of species 2 that eluted

Particle_1_elution_h $=$ [Particle_1_elution_h

z12(np1_eluted)]; $\%$ Array of eluted particle heights

Particlēe2_elution_h $=$ [Particle_2_elution_h

z12(np2_eluted)]; \% Array of eluted particle heights

np1_col $=$ length $(z 12(z 12($ np1_eluted $)>(.5 * h))) ; \quad$ Indices of

species 1 collected

np2_col $=$ length $(z 12(z 12($ np2_eluted $)<(.5 * h))) ; \quad$ Indices of

species 2 collected 
np1_eluted $=$ length $(\mathrm{np} 1$ _eluted $) ;$ Number of particle 1 eluted np2_eluted $=$ length $(\mathrm{np} 2$-eluted $) ;$ \% Number of particle 2 eluted tot_np1_eluted $=$ tot_np1_eluted + npl_eluted; $\%$ Total number of species 1 eluted

tot_np2_eluted = tot_np2_eluted + np2_eluted; $\%$ Total number of species 2 eluted

np1_collection $=$ npl_collection + np1_col; $\%$ Total number of species 1 collected

np2_collection $=$ np2_collection + np2_col; $\%$ Total number of species 2 collected

tot_np1 = tot_np1 + np1_eluted;

tot_np2 = tot_np2 + np2_eluted;

\% Particle Re-entry for continuous flow

$\mathrm{x} 12$ (eluted) $=0$;

\% Particle re-entry for continuous flow

y12(eluted) $=\mathrm{w}^{*}$ rand $(1$, length(eluted)); $\% \mathrm{y}$-location for reinitialized particles

$\mathrm{z} 12($ eluted $)=(\mathrm{h} / 8) *(7+$ rand $(1$, length $($ eluted $))) ;$-location

for re-initialized particles

end

을 Evaluation

tot_np1=tot_np1-np1 \% Total number of particle 1 injected

tot_np2=tot_np2-np2 \% Total number of particle 2 injected

Yield_1 = np1_collection/tot_np1 $\quad$ Species 1 Yield

Yield_2 = np2_collection/tot_np2 $\%$ Species 2 Yield

Purity_1 = np1_collection/(np1_collection+tot_np2_elutednp2_collection) \% Species 1 Purity

Purity_2 = np2_collection/(np2_collection+tot_np1_elutednp1_collection) \% Species 2 Purity

p1_avg_h $=$ mean(Particle_1_elution_h) $\quad$ o Average height of species 1

p2_avg_h $=$ mean (Particle_2_elution_h) $\%$ Average height of

species 2

st_dev_1 = std(Particle_1_elution_h) $\quad$ o Standard deviation

of species 1 heights

st_dev_2 = std(Particle_2_elution_h) $\quad$ Standard deviation

of species 1 heights

suggested_separation_cutoff $=((\mathrm{p} 1$ _avg_h-p2_avg_h-st_dev_1st_dev_2) $/ 2)+p 2$ avg_h + st_dev_2 


\section{A.2 Pozrikidiz Velocity Function}

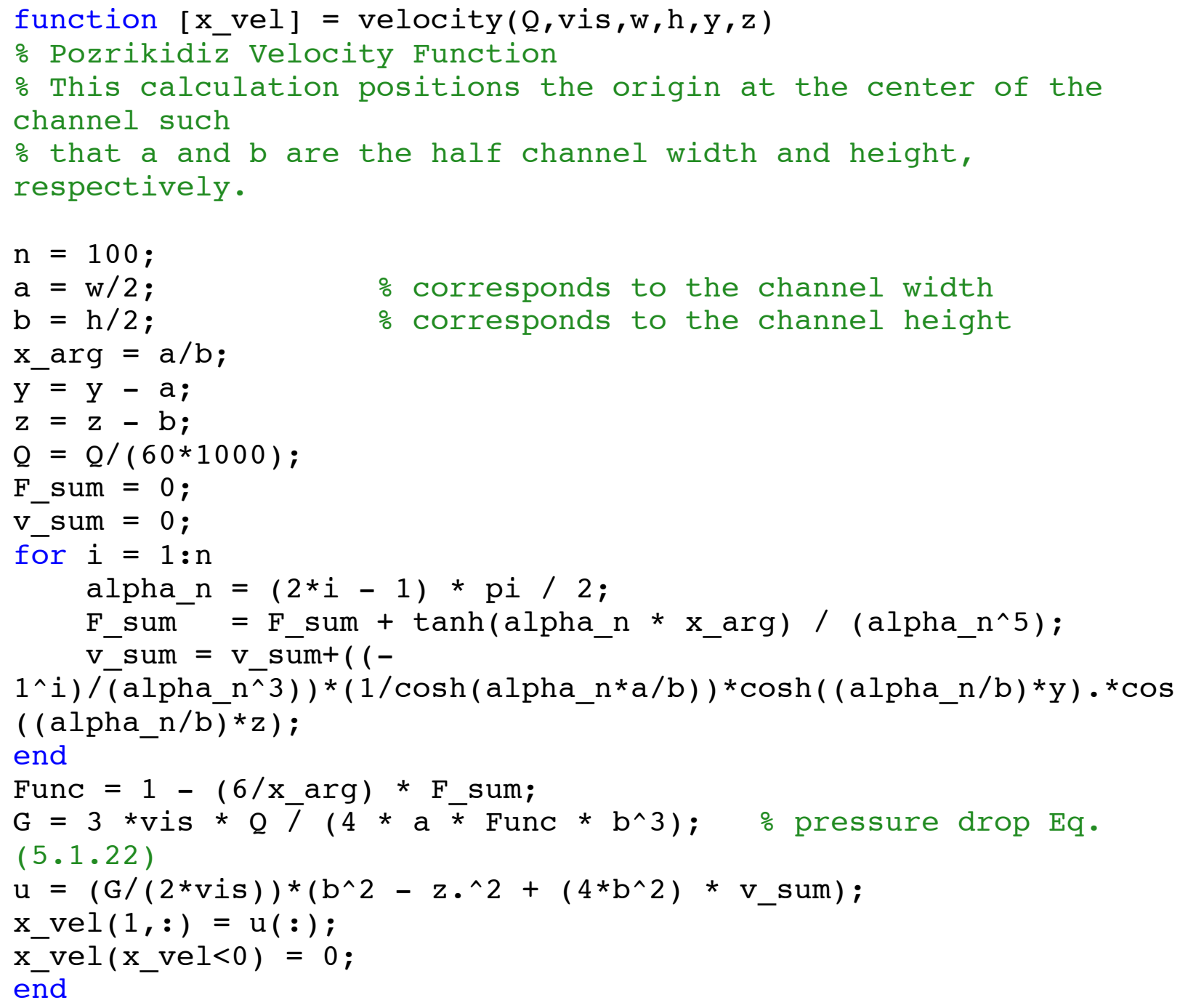

\section{A.3 Bruus Velocity Function}

function [x_vel] = Vel_Function $(Q, w, h, y, z)$

\% Velocity Function from Bruus, Mortensen, Lima "In vitro blood flow in a

\% rectangular PDMS microchannel: experimental observations using

a confocal

\% micro-PIV system"

$\mathrm{n}=100$;

conversion $=1 /(60 * 1000) ; \% \mathrm{~m}^{\wedge} 3 /(\mathrm{s} *$ liters $)$

$\mathrm{Q}=\mathrm{Q} *$ conversion; $\%$ conversion from liters $/ \mathrm{min}$ to $\mathrm{m}^{\wedge} 3 / \mathrm{s}$

num $=0$;

denom $=0$; 


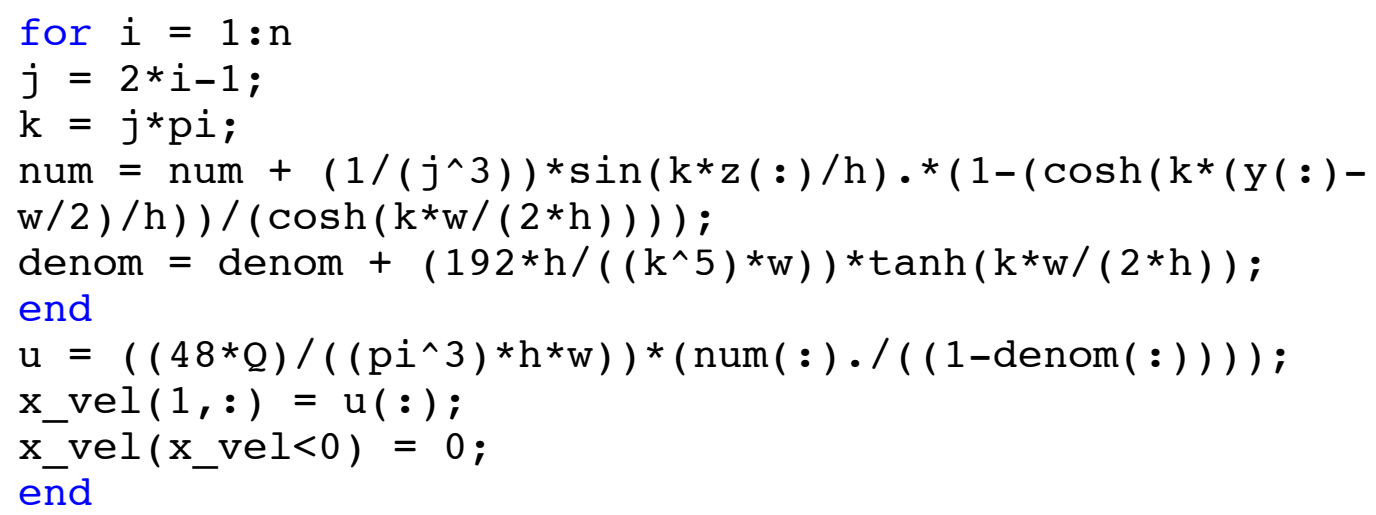

\section{A.4 Distance Calculations}

function [ $r$ c ri ci D] = Collision_Detection $(x, y, z, r p$, lambda_D) $\circ$ Finds the pairwise distance^ 2 between all particles

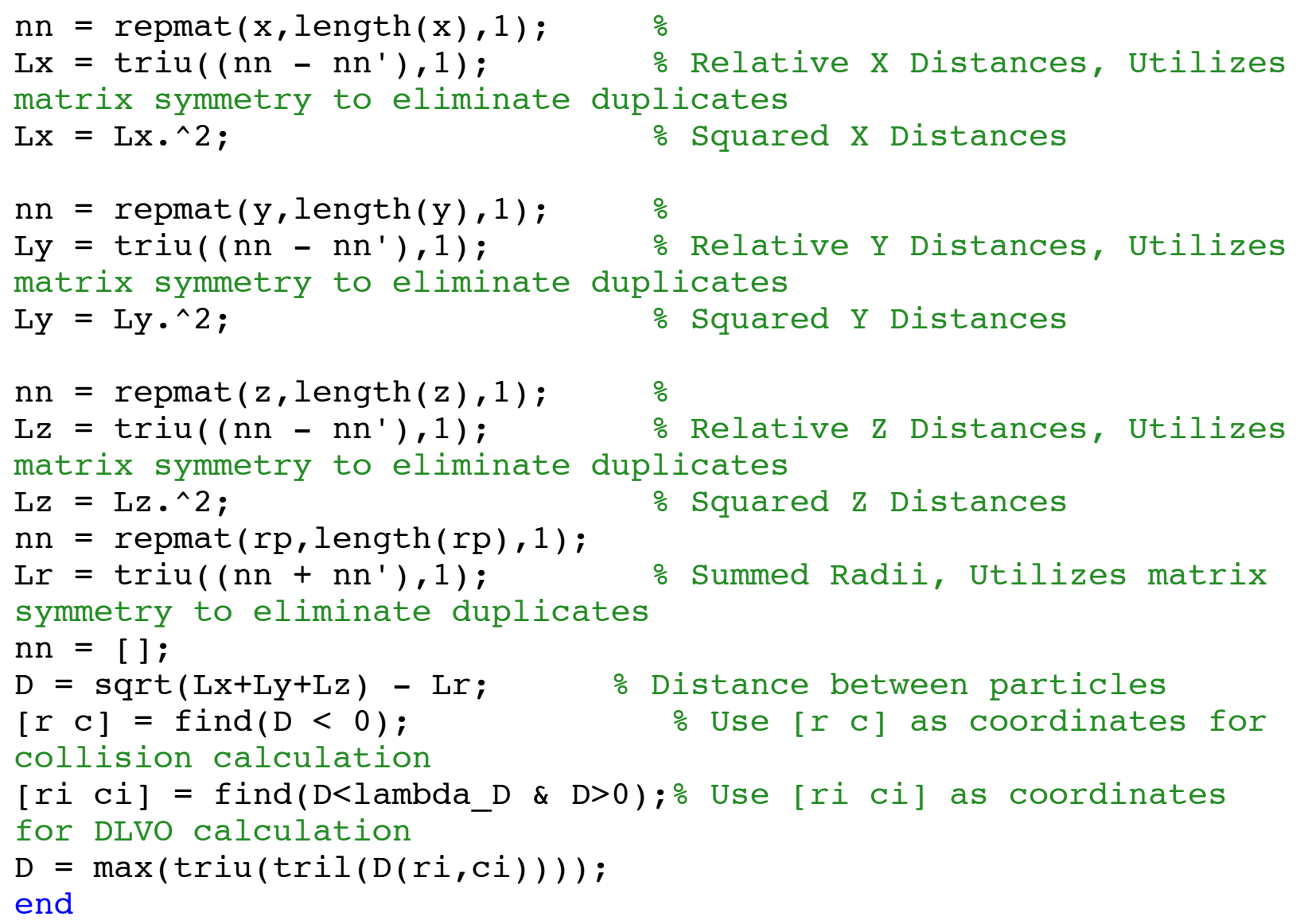




\section{A.5 Kinetic Collision Response}

function $[x, y, z]=\operatorname{Collision}(x, y, z, d x, d y, d z, m, r, c, d t$, eta, rad)

\% For the Collision response, [ $r$ c $]$ correspond to particles a $\& b$,

\% respectively. [ $r$ c] serve as the indices for the location and velocity

$\%$ of $a \& b$.

$\mathrm{U}=[\mathrm{dx} \cdot / \mathrm{dt} ; \mathrm{dy} \cdot / \mathrm{dt} ; \mathrm{dz} \cdot / \mathrm{dt}] ;$ \% Initial Particle Velocities

$\mathrm{Uab}=\operatorname{zeros}(3$, length $(\mathrm{x}))$;

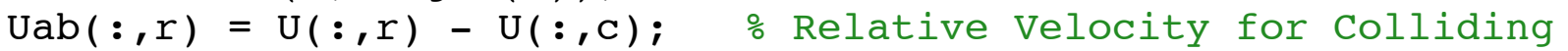

Particles a \& b

$\operatorname{Uab}(:, \mathrm{c})=\operatorname{Uab}(:, \mathrm{r}) ; \quad$ o Relative Velocity for Colliding

Particles a \& b

$\mathrm{Va}=\operatorname{zeros}(3$, length $(\mathrm{x})) ; \quad$ Final Velocity Matrix, particle a

$\mathrm{Vb}=\operatorname{zeros}(3$, length $(\mathrm{x})) ; \quad$ Final Velocity Matrix, particle $\mathrm{b}$

$\mathrm{R}=[\mathrm{x} ; \mathrm{y} ; \mathrm{z}]$;

\% Position vector of particles

$\mathrm{u}_{-} \mathrm{n}=\operatorname{zeros}(3$, length $(\mathrm{x}))$;

$u_{-} \mathrm{n}(:, r)=(\mathrm{R}(:, r)-\mathrm{R}(:, \mathrm{c})) \cdot / \operatorname{repmat}(\operatorname{sqrt}(\operatorname{sum}((\mathrm{R}(:, r)-$

$\left.\left.\left.\mathrm{R}(\mathbf{:}, \mathrm{C})) \cdot{ }^{\wedge} 2\right)\right), 3,1\right)$; o Unit Normal Vector from Particle a to b u_n $(:, C)=u_{-} n(:, r)$;

Normal Vector from Particle a to b

dot_r $=\operatorname{zeros}(1$, length $(\mathrm{x}))$;

dot_c $=\operatorname{zeros}(1$, length $(\mathrm{x}))$;

dot_r(r) $\operatorname{sum}\left(\operatorname{Uab}(:, r) . * u \_n(:, r)\right)$; $\%$ Relative Velocity

component along Unit Normal

dot_c(c) $=\operatorname{sum}\left(\operatorname{Uab}(:, \mathbf{c}) \cdot{ }^{*} u_{-} \mathrm{n}(:, \mathbf{c})\right)$; $\%$ Relative Velocity

component along Unit Norma $\bar{l}$

$\mathrm{Va}(:, r)=\mathrm{U}(:, r)-$

$(1+$ eta $) * \operatorname{repmat}\left((m(c) . /(m(r)+m(c))) \cdot *\left(\operatorname{dot} \_r(r)\right), 3,1\right) \cdot * u \_n(:, r)$;

Particle a

o Final Velocity of

$\mathrm{Vb}(:, \mathrm{C})=\mathrm{U}(:, \mathrm{C})+$

$(1+e t a) * r e p m a t\left((m(r) . /(m(r)+m(c))) . *\left(\operatorname{dot} \_c(c)\right), 3,1\right) . * u \_n(:, c)$;

Particle b

o Final Velocity of 


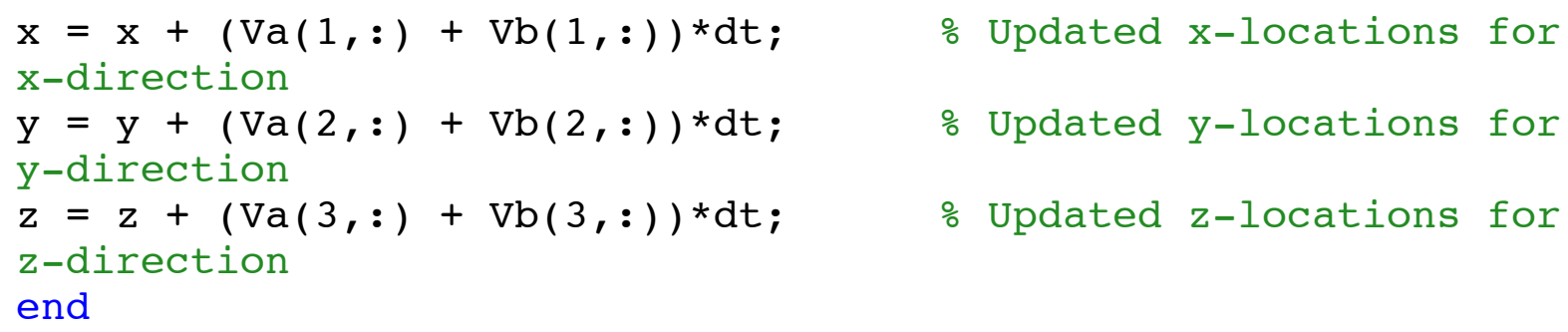

\section{A.6 DLVO Interactions}

function $\left[\mathrm{X}, \mathrm{Y}, \mathrm{Z}, \mathrm{V} \_\mathrm{r}, \mathrm{V}{ }_{-} \mathrm{C}\right]=\mathrm{DLVO}\left(\mathrm{X}, \mathrm{Y}, \mathrm{Z}, \mathrm{R}, \mathrm{M}, \mathrm{r}, \mathrm{C}, \mathrm{A} \_\mathrm{H}, \mathrm{D}\right.$, gamma, epsilon, $k, \mathrm{~K} \bar{b}, \mathrm{~T}, \mathrm{z}$, elec, $\mathrm{dt}$ )

\% $r$ and $c$ are the row and column indices of interaciting

particles from the

\% distance calculations

for $i=1:$ length $(r)$

$r$ vect $=[\mathrm{X}(\mathrm{r}(\mathrm{i}))-\mathrm{X}(\mathrm{C}(\mathrm{i})) ; \mathrm{Y}(\mathrm{r}(\mathrm{i}))-\mathrm{Y}(\mathrm{c}(\mathrm{i})) ; \mathrm{Z}(\mathrm{r}(\mathrm{i}))-\mathrm{Z}(\mathrm{c}(\mathrm{i}))]$;

Vector between interacting particles

$r$ mag $=\operatorname{sqrt}\left(\operatorname{sum}\left(\left(r \_\right.\right.\right.$vect.^$\left.\left.\left.{ }^{\wedge} 2\right), 1\right)\right)$;

u_r_vect $(:, i)=r_{-}$vect/r_mag;

u_c_vect $(:, i)=-\bar{r} \_$vect $/ \bar{r} \_$mag;

end

$\mathrm{DA}=\left(\mathrm{R}(\mathrm{r}) \cdot{ }^{\mathrm{R}}(\mathrm{C})\right) \cdot /(\mathrm{R}(\mathrm{r})+\mathrm{R}(\mathrm{C})) ; \quad$ \% Derjaquin Approximation

for Sphere-Sphere

$\mathrm{W} \_\mathrm{vdW}=-\left(\mathrm{A} \_\mathrm{H} / 6\right) *(\mathrm{DA} . / \mathrm{D})$; $\%$ Van der Waals Attractive Force

$\mathrm{W}$ _rep $=$

$6 \overline{4} * \mathrm{pi} *$ epsilon* $((\mathrm{Kb} * \mathrm{~T} /(\mathrm{z} * \mathrm{elec})) \wedge 2) * g a m m a(r) . * g a m m a(\mathrm{c}) \cdot * \mathrm{DA} . * \exp (-$ $\mathrm{k} * \mathrm{D})$;

$\mathrm{W} \_\mathrm{DLVO}=\mathrm{W} \_\mathrm{vdW}+\mathrm{W}$ rep;

$\mathrm{abs}$ _DLVO $=-\operatorname{abs}\left(\mathrm{W} \_\mathrm{DLVO}\right)$;

$v_{-} r^{-}=\operatorname{zeros}(3$, length $(X))$;

$\mathrm{v}_{-} \mathrm{C}=\operatorname{zeros}(3$, length $(\mathrm{X}))$;

$v_{-} r(:, r)=$

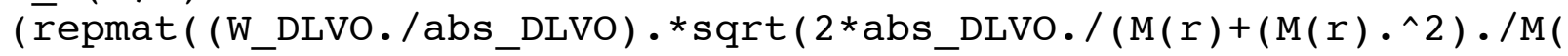
C) ) $), 3,1)) \cdot * u_{-} r_{-} v e c t ;$

$\mathrm{V}_{-} \mathrm{C}(:, \mathrm{C})=$

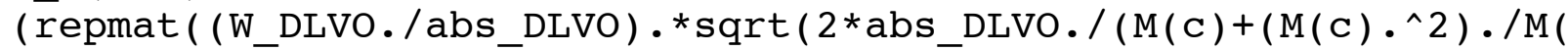
r) ) ) $, 3,1)) \cdot *$ u_c_vect;

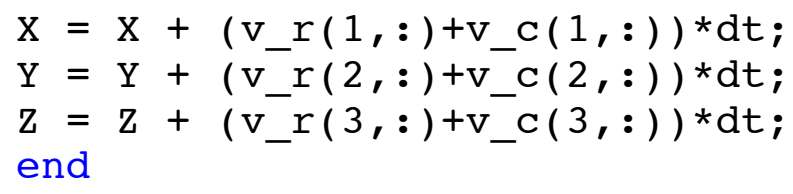

